\title{
Pressure-Induced Phase-Transition Sequence In Cof 2: An Experimental And First-Principles Study On The Crystal, Vibrational, And Electronic Properties
}

\author{
J.A. Barreda-Argüeso \\ S. López-Moreno \\ M. N. Sanz-Ortiz \\ A. H. Romero
}

Follow this and additional works at: https://researchrepository.wvu.edu/faculty_publications

\section{Digital Commons Citation}

Barreda-Argüeso, J. A.; López-Moreno, S.; Sanz-Ortiz, M. N.; and Romero, A. H., "Pressure-Induced Phase-Transition Sequence In Cof 2 : An Experimental And First-Principles Study On The Crystal, Vibrational, And Electronic Properties" (2013). Faculty Scholarship. 994.

https://researchrepository.wvu.edu/faculty_publications/994 


\title{
Pressure-induced phase-transition sequence in $\mathrm{CoF}_{2}$ : An experimental and first-principles study on the crystal, vibrational, and electronic properties
}

\author{
J. A. Barreda-Argüeso, ${ }^{1}$ S. López-Moreno,,${ }^{2, *}$ M. N. Sanz-Ortiz, ${ }^{1}$ F. Aguado, ${ }^{1}$ R. Valiente, ${ }^{3}$ \\ J. González, ${ }^{1}$ F. Rodríguez, ${ }^{1}$ A. H. Romero, ${ }^{4}$ A. Muñoz, ${ }^{5}$ L. Nataf, ${ }^{6}$ and F. Baudelet ${ }^{6}$ \\ ${ }^{1}$ DCITIMAC, MALTA CONSOLIDER Team, Universidad de Cantabria, 39005 Santander, Spain \\ ${ }^{2}$ Escuela Superior Cd. Sahagún Universidad Autónoma del Estado de Hidalgo, \\ Carretera Cd. Sahagún-Otumba s/n. 43990, Hidalgo, México \\ ${ }^{3}$ Departamento de Física Aplicada, MALTA CONSOLIDER Team, Universidad de Cantabria, 39005 Santander, Spain \\ ${ }^{4}$ Physics Department, West Virginia University, Morgantown, West Virginia, 26506-6315, USA \\ ${ }^{5}$ Departamento de Física Fundamental II, Instituto de Materiales y Nanotecnología Universidad de La Laguna, \\ La Laguna 38205, Tenerife, Spain \\ ${ }^{6}$ Synchrotron SOLEIL, L'Orme des Merisiers, St Aubin BP48, 91192 Gif-sur-Yvette cedex, France
}

(Received 16 September 2013; published 19 December 2013)

\begin{abstract}
We report a complete structural study of $\mathrm{CoF}_{2}$ under pressure. Its crystal structure and vibrational and electronic properties have been studied both theoretically and experimentally using first-principles density functional theory (DFT) methods, $\mathrm{x}$-ray diffraction, $\mathrm{x}$-ray absorption at Co $K$-edge experiments, Raman spectroscopy, and optical absorption in the 0-80 GPa range. We have determined the structural phase-transition sequence in $\mathrm{CoF}_{2}$ and corresponding transition pressures. The results are similar to other transition-metal difluorides such as $\mathrm{FeF}_{2}$ but different to $\mathrm{ZnF}_{2}$ and $\mathrm{MgF}_{2}$, despite that the $\mathrm{Co}^{2+}$ size (ionic radius) is similar to $\mathrm{Zn}^{2+}$ and $\mathrm{Mg}^{2+}$. We found that the complete phase-transition sequence is tetragonal rutile $\left(P 4_{2} / \mathrm{mnm}\right) \rightarrow \mathrm{CaCl}_{2}$ type (orthorhombic Pnnm) $\rightarrow$ distorted $\mathrm{PdF}_{2}$ (orthorhombic $P b c a)+\mathrm{PdF}_{2}$ (cubic $P a \overline{3}$ ) in coexistence $\rightarrow$ fluorite (cubic $F m \overline{3} m$ ) $\rightarrow$ cotunnite (orthorhombic Pnma). It was observed that the structural phase transition to the fluorite at $15 \mathrm{GPa}$ involves a drastic change of coordination from sixfold octahedral to eightfold cubic with important modifications in the vibrational and electronic properties. We show that the stabilization of this high-pressure cubic phase is possible under nonhydrostatic conditions since ideal hydrostaticity would stabilize the distorted-fluorite structure (tetragonal $I 4 / \mathrm{mmm}$ ) instead. Although the first rutile $\rightarrow \mathrm{CaCl}_{2}$-type second-order phase transition is subtle by Raman spectroscopy, it was possible to define it through the broadening of the $E_{g}$ Raman mode which is split in the $\mathrm{CaCl}_{2}$-type phase. First-principles DFT calculations are in fair agreement with the experimental Raman mode frequencies, thus providing an accurate description for all vibrational modes and elastic properties of $\mathrm{CoF}_{2}$ as a function of pressure.
\end{abstract}

DOI: 10.1103/PhysRevB.88.214108

PACS number(s): 61.05.-a, 61.50.Ks, 63.20.dk, 71.15.Mb

\section{INTRODUCTION}

Pressure-induced structural phase transitions of transitionmetal $(T M)$ dihalides $T M X_{2}(X: \mathrm{Cl}, \mathrm{Br}, \mathrm{F})$ have received considerable attention over the past 30 years due to their ample and subtle polymorphism. ${ }^{1-14}$ Most transformations yield a high variety of energetically equivalent structures, which are characterized by an increase of the $T M$ coordination number upon compression. Due to their simple composition and bonding, the phase-transition sequence shows some common features associated with the coordination polyhedra, providing a general polymorphic description for these transformations, which results in the interest in geophysics as well as in materials science. In particular, this behavior has enormous implications as the electronic properties are substantially modified with the change of $T M$ coordination, but also in geophysics as the ample polymorphism attained in the Earth, where $\mathrm{SiO}_{2}$ quartz is an example of this behavior. ${ }^{15-17}$ The ambient pressure quartz-coesite structure, which is characterized by a three-dimensional (3D) network of O-sharing $\mathrm{SiO}_{4}^{4-}$ tetrahedra, transforms to the stishovite phase at high pressure, which is the high-pressure polymorph of the rutile structure. This last phase has called a large attention since it appears in the Earth's lower mantle. ${ }^{15}$ In spite of the significant difference between $T M-\mathrm{F}$ and Si-O bonds, many $T M \mathrm{~F}_{2}$ exhibit a similar H-P-T sequence, although slight deviations can appear depending on the nature of the TM. Pioneering works in $\mathrm{MnF}_{2}$ and $\mathrm{CoF}_{2}$ (Refs. 1 and 2) showed that these compounds transform from rutile to fluorite at high pressure. However, the development of $\mathrm{x}$-ray diffraction (XRD) techniques devoted to high pressure at synchrotron facilities allowed us to unveil slight structural differences with respect to the simplest transition sequence, which led to the description of $\mathrm{ZnF}_{2}$ and $\mathrm{MgF}_{2}$ pressure behavior in terms of a phase-transition sequence. For nonmagnetic difluoride compounds such as $\mathrm{MgF}_{2}$ (Refs. 6 and 8) and $\mathrm{ZnF}_{2},{ }^{9}$ the sequence of structural phase transitions is rutile $\left(P 4_{2} / m n m\right) \rightarrow \mathrm{CaCl}_{2}$ type $(P n n m) \rightarrow \mathrm{PdF}_{2}$ type $(P a \overline{3})$. While the rutile $\rightarrow \mathrm{CaCl}_{2}$-type phase transition is of the second order and it is properly ferroelastic, ${ }^{6,7}$ the phase-transition $\mathrm{CaCl}_{2}$ type $\rightarrow \mathrm{PdF}_{2}$-type is a first-order transition and involves a volume reduction of $6 \%$. For these transitions there is no change in the coordination number for the $T M$ atoms, such that in the fluorite-distorted $\mathrm{PdF}_{2}$-type phase, the cation coordination is $6+2$ instead of $8 .{ }^{9}$ On the other hand, according to Ref. 2, TM difluorides such as $\mathrm{MnF}_{2}, \mathrm{CoF}_{2}$, and $\mathrm{NiF}_{2}$ exhibit a quite different sequence: rutile $\rightarrow$ orthorhombic structure $\rightarrow$ $\mathrm{DF} \rightarrow \mathrm{PbCl}_{2}$-type structure, rutile $\rightarrow \mathrm{DF} \rightarrow$ hexagonal structure, and rutile $\rightarrow$ orthorhombic structure $\rightarrow$ DF, respectively, where DF refers to a distorted fluorite phase. However, the lack 
of accurate experimental data on these compounds precludes any attempt to generalize this transition sequence and to establish the main driving forces leading to a given sequence within difluorides. In this way, accurate density functional theory (DFT) ab initio studies performed in $\mathrm{FeF}_{2}$ have clearly demonstrated this problem is subtle, as evidenced by the possibility of phase coexistence associated with phases where the free energy only differ by a few meV. ${ }^{10}$ Moreover, these calculations reveal that nonhydrostatic conditions can stabilize other phases more symmetric than those stabilized under hydrostatic conditions.

These important constraints make the experimental study of $T M \mathrm{~F}_{2}$ complex and difficult due likely to multiphase formation. Therefore, the use of XRD with complementary techniques such as optical absorption, x-ray absorption, and Raman spectroscopy can be decisive to draw an adequate characterization of the phase-transition sequence.

Here, we investigate the phase-transition sequence in $\mathrm{CoF}_{2}$ as a function of pressure from the rutile to the cotunnite structure (0-80 GPa range) using the above-mentioned experimental techniques and accurate $a b$ initio calculations. From a stereochemistry view point, the interest for studying $\mathrm{CoF}_{2}$ is twofold: (1) as far as the ionic radius is concerned $\left(R_{\mathrm{Co}^{2+}}=\right.$ $0.75 \AA)$, the phase-transition sequence should be similar to $\mathrm{ZnF}_{2}$ and $\mathrm{MgF}_{2}\left(R_{\mathrm{Zn}^{2+}}=0.74 \AA ; R_{\mathrm{Mg}^{2+}}=0.72 \AA\right)$ rather than $\mathrm{FeF}_{2}\left(R_{\mathrm{Fe}^{2+}}=0.78 \AA\right) ;{ }^{18}(2)$ as $\mathrm{Fe}^{2+}\left(3 d^{6}\right), \mathrm{Co}^{2+}$ has an open $d$-orbital configuration $\left(3 d^{7}\right)$, and additional bonding due to $d$ electrons should favor a similar behavior to $\mathrm{FeF}_{2} .{ }^{19}$ Therefore, investigation on $\mathrm{CoF}_{2}$ can be crucial to clarify which of the interactions eventually governs the phase-transition sequence. Interestingly, this study will particularly focus on the possible existence of the rutile $\rightarrow \mathrm{CaCl}_{2}$-type second-order phase transition, which is properly ferroelastic, ${ }^{6,9}$ and difficult to detect experimentally by Raman spectroscopy.

The paper is organized as follows: In Secs. II and III, we give a detailed description of the experimental and computational details. The experimental and theoretical results are in Sec. IV, where we show the experimental XRD studies in Sec. IV A, the Raman measurements in Sec. IV B, XANES and EXAFS data in Sec. IV C, and the optical absorption measurements in Sec. IVD. In Sec. IVE, we analyze the theoretical predictions for the phase-transition sequence of $\mathrm{CoF}_{2}$ under ideal hydrostatic conditions. Finally, the summary and conclusions of this work are presented in Sec. V.

\section{EXPERIMENTAL DETAILS}

Float-zone single crystals of $\mathrm{CoF}_{2}$ growth by Bridgman were used in present experiments. Suitable parallelepiped samples of $\mathrm{CoF}_{2}\left(90 \times 90 \times 20 \mu \mathrm{m}^{3}\right)$ cut perpendicular to the tetragonal $c$ axis were obtained from the same crystal rod and used for optical absorption and Raman spectroscopy under high-pressure conditions. Unpolarized micro-Raman scattering measurements were performed in a triple monochromator Horiba-Jobin-Yvon T64000 spectrometer in subtractive mode backscattering configuration, equipped with liquid-nitrogencooled CCD detector. The 514.5-nm and 647-nm lines of an $\mathrm{Ar}^{+}-\mathrm{Kr}^{+}$laser were focused on the sample with a $20 \times$ objective for micro-Raman, and the laser power was kept below $40 \mathrm{~mW}$ in order to avoid heating effects. The laser spot was $20 \mu \mathrm{m}$ in diameter and the spectral resolution was better than $1 \mathrm{~cm}^{-1}$. The Raman technique was used to check the sample structure through the characteristic first-order modes $\left(A_{1 g}\right.$ and $E_{g}$ in rutile-type structure) as well as to determine structural phase-transition pressures.

Optical absorption under high-pressure conditions was performed on a prototype fiber-optics microscope equipped with two $25 \times$ reflecting objectives mounted on two independent $x-y-z$ translational stages for the microphocus beam, and the collector objective and a third independent $x-y-z$ translational stage for the diamond anvil cell (DAC) holder. Optical absorption, luminescence, and imaging data were obtained simultaneously with the same device. ${ }^{20}$ Spectra in the UV-VIS and NIR were attained with specially designed Ocean

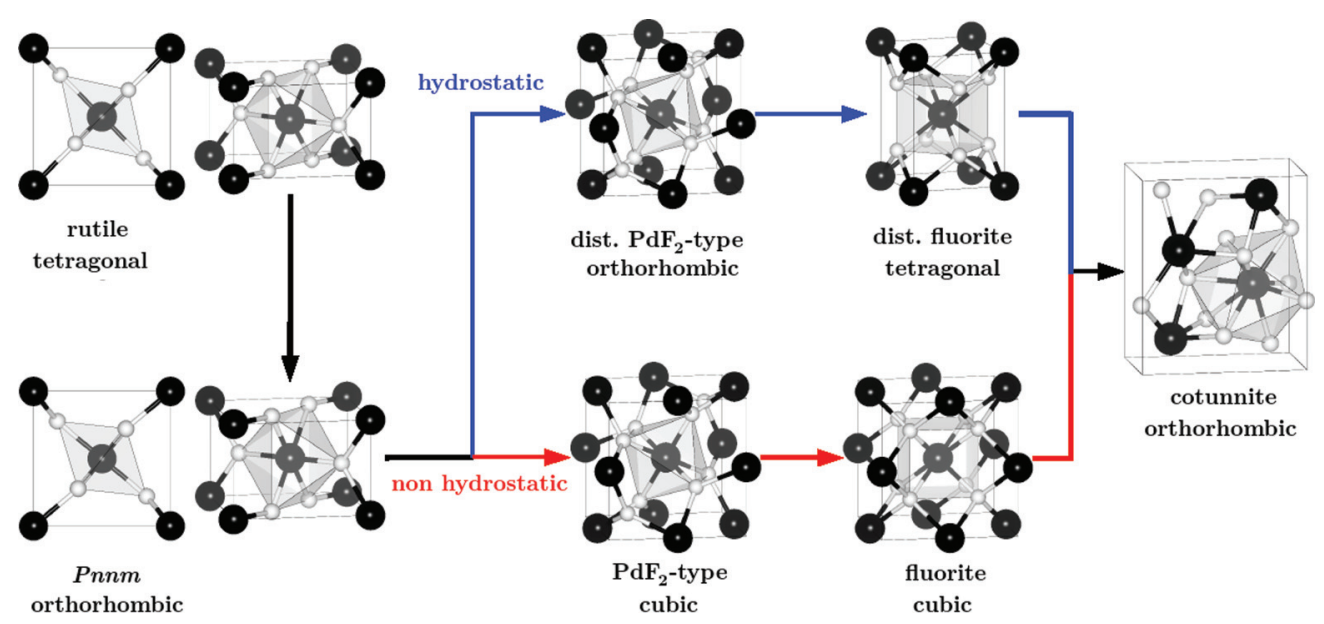

FIG. 1. (Color online) First-principles DFT calculations of the structural phase-transition sequence in $\mathrm{CoF}_{2}$ under ideal hydrostatic and nonhydrostatic conditions. The lattice cells show the $\mathrm{F}^{-}$coordination polyhedron around the $\mathrm{Co}^{2+}$ ion in each phase. In the nonhydrostatic calculations, there is a difference of $\approx 0.3 \mathrm{GPa}$ in the values of stress tensor in $x, y$, and $z$ components, whereas the energy difference in the enthalpy among $\mathrm{PdF}_{2}$ and distorted (dist.) $\mathrm{PdF}_{2}$ (dist. fluorite and fluorite) phases is less than $0.3 \mathrm{meV} /$ f.u. The graphics where performed with the VESTA program (Ref. 24). 
Optics USB 2000 and NIRQUEST 512 monochromators using $\mathrm{Si}-$ and InGaAs-CCD detectors, respectively.

Hydrostatic pressure experiments in the 0-80 GPa range were carried out on a membrane-type diamond anvil cell (DAC) and a Boehler-Almax DAC for high-pressure experiments. 200- $\mu \mathrm{m}$-thickness Inconel gaskets were preindented and suitable $200-\mu \mathrm{m}$-diameter holes were perforated with a Betsa motorized electrical discharge machine. The DAC was loaded with a suitable single crystal and ruby microspheres (10 $\mu \mathrm{m}$ diameter) using silicon oil as pressure-transmitting medium.

XRD experiments under pressure were performed at the MSPD beamline in the ALBA Synchrotron Facility, Spain, using monochromatic radiation $(\lambda=0.4246 \AA)$ and a twodimensional (2D) image-plate detector, in combination with a Boelher-Almax DAC with $700-\mu \mathrm{m}$ and $350-\mu \mathrm{m}$ culet diamonds to cover accurately the $0-20 \mathrm{GPa}$ range. X-ray absorption measurements under pressure were obtained in the ODE: a new beam line for high-pressure XAS and XMCD studies at SOLEIL, ${ }^{21}$ using energy dispersive setup with white beam around the Co $K$ edge $(E=7.73 \mathrm{keV})$ and a membrane-type DAC.

In all experiments we used silicone oil as pressuretransmitting media and, additionally, we also employed paraffin in Raman and optical absorption measurements. The pressure and temperature were calibrated from the ruby PL through the $R_{1}$ and $R_{2}$ peak shifts and their relative intensity, respectively. ${ }^{22,23}$

\section{COMPUTATIONAL DETAILS}

Total-energy calculations were performed within the framework of the DFT and the projector-augmented wave ${ }^{25,26}$ (PAW) method as implemented in the Vienna $a b$ initio simulation package (VASP). ${ }^{27-30}$ We used a plane-wave energy cutoff of $520 \mathrm{eV}$ to ensure a high precision in all our calculations. The exchange and correlation energy was described within the generalized gradient approximation (GGA) in the PerdewBurke-Ernzerhof $^{31}$ (PBE) prescription. The GGA $+U$ method was used to account for the strong correlation between the electrons in the Co $d$ shell, on the basis of Dudarev's method. ${ }^{32}$ In this method, the onsite Coulomb interaction $U$ and onsite exchange interaction $J^{\mathrm{H}}$ were treated together as $U_{\text {eff }}=U-J^{\mathrm{H}}$. For our GGA $+U$ calculations, we chose $U=6 \mathrm{eV}$ and $J^{\mathrm{H}}=1 \mathrm{eV}$ for the Co atom. These values of $U$ and $J^{\mathrm{H}}$ were used previously with relative success in other cobalt-based compounds such as $\mathrm{CoWO}_{4} \cdot{ }^{33}$ To ensure the quality of the chosen $U_{\text {eff }}$, we compared the electronic and structural parameters for the rutile structure with reported data in the literature, where good agreement was found. To further test the effect of $U_{\text {eff }}$, we calculated the dependence of the pressure at which the structural transition occurs for the first phase transitions [see Fig. 1(c)] as a function of the value of $U_{\text {eff }}$. As the $U_{\text {eff }}$ changes from 2.5 to $6.0 \mathrm{eV}$, the change in the transition pressure is less than $0.3 \mathrm{GPa}$. This indicates that the structural transitions are not very sensitive to the choice of $U_{\text {eff }}$ but its presence is important to describe correctly the electronic localization and magnetic properties.

The Monkhorst-Pack scheme was employed for the Brillouin-zone (BZ) integrations ${ }^{34}$ with a mesh $4 \times 4 \times 6$,
$4 \times 4 \times 6,4 \times 4 \times 4,4 \times 4 \times 4$, and $4 \times 6 \times 3$, which corresponds a set of $9,12,8,6$, and 12 special $k$ points in the irreducible BZ for rutile (SG: $P 4_{2} / m n m$, No. $136, Z=2$ ), $\mathrm{CaCl}_{2}$ type (SG:Pnnm, No. 58, Z=2), distorted $\mathrm{PdF}_{2}$ type (SG:Pbca, No. $61, Z=4$ ), tetragonal (distorted fluorite, SG: $I 4 / \mathrm{mmm}$, No. $139, Z=2$ ), and cotunnite (SG:Pnma, No. 62, $Z=4$ ), respectively. For the additional structures considered in the high-pressure regime, we used the mesh most suitable for each case. In the relaxed equilibrium configuration, the forces were less than $1 \mathrm{meV} / \AA$ per atom in each of the Cartesian directions. The highly converged results on forces were required for the calculations of the dynamical matrix using the direct force constant approach (or supercell method). ${ }^{35}$
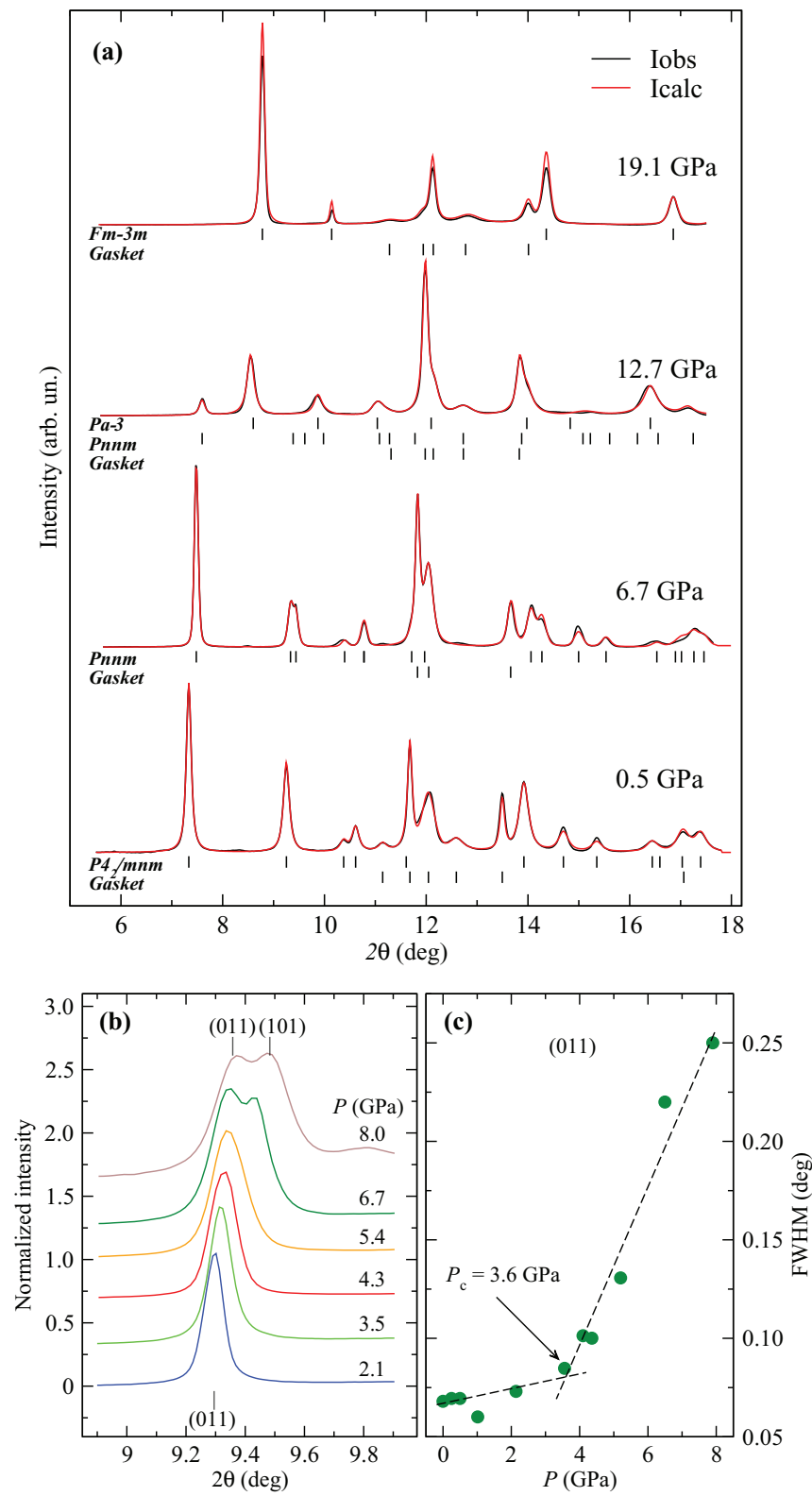

FIG. 2. (Color online) (a) Selected experimental x-ray diffraction patterns (Iobs) of $\mathrm{CoF}_{2}$ at representative pressures, and calculated (Icalc) diffraction patterns with the indicated crystal phases. (b) and (c) show the variation of the (011) peak width with pressure. The peak broadening and further split above $3.6 \mathrm{GPa}$ reveals the first rutile- $P$ nnm phase transition in $\mathrm{CoF}_{2}$ at $3.6 \mathrm{GPa}$. 
TABLE I. Experimental (room temperature) and theoretical $(T=0 \mathrm{~K}) \mathrm{CoF}_{2}$ lattice parameters and Wyckoff positions (WPs) for rutile (space group, $\mathrm{SG}: P 4_{2} / \mathrm{mnm}$ ) and high-pressure phases: $\mathrm{CaCl}_{2}$-type structure (SG: $\left.P n n m\right)$, $\mathrm{PdF}_{2}$ type (SG: $\left.P a \overline{3}\right)$, distorted PdF 2 type (SG: $P b c a$ ), fluorite (SG: $F m \overline{3} \mathrm{~m}$ ), distorted fluorite (SG: $I 4 / \mathrm{mmm}$ ), and cotunnite (SG:Pnma) at the corresponding pressure $P . a, b$, and $c$ are the lattice parameters, $V$ is the equilibrium volume at the respective pressure, $\left\langle d_{\mathrm{Co}-\mathrm{F}}\right\rangle$ is the interatomic bond distance among Co and $\mathrm{F}, Z$ is the number of formula units (f.u.) in the unit cell, $B_{0}$ the bulk modulus, $B_{0}{ }^{\prime}$ the first derivative of the bulk modulus with respect to pressure, $\mu_{\mathrm{Co}}$ the magnetic moment of Co per f.u., and the optimized WPs from each structure. Standard deviations are given in parentheses.

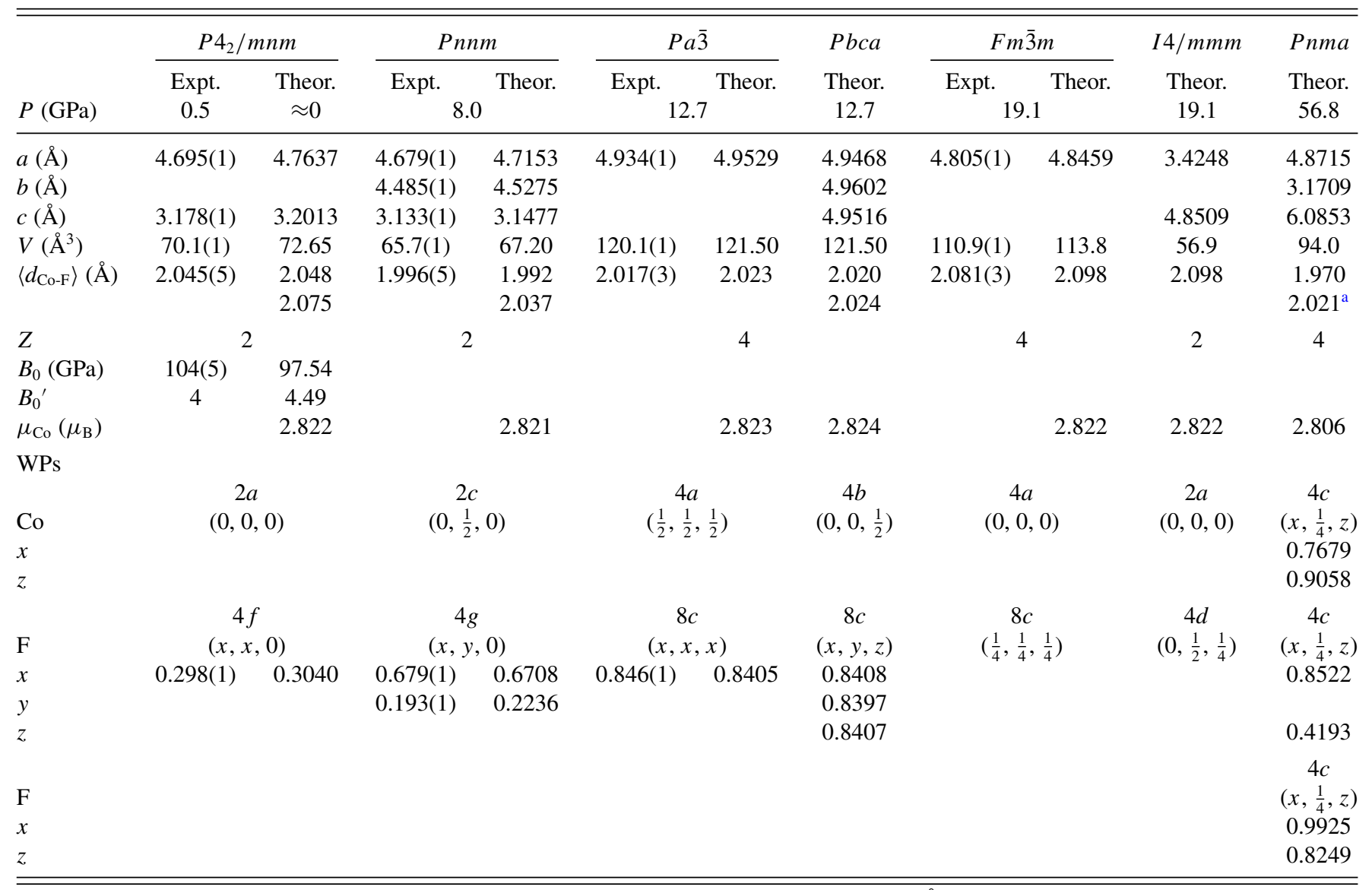

${ }^{\mathrm{a}}$ For cotunnite phase, there are five $d_{\mathrm{Co-F}}$ distances: $1.9704,1.9971,2.0208,2.0267$, and $2.0293 \AA$.

\section{RESULTS}

\section{A. XRD studies}

At low temperature and ambient conditions, cobalt (II) fluoride $\left(\mathrm{CoF}_{2}\right)$ crystallizes in the tetragonal rutile structure with the space group $P 4_{2} / m n m$ ( $D_{4 h}^{14}$ in the Schoenflies notation) and with two formula units per conventional cell (see Fig. 1). The rutile structure is composed of alternating neighboring $\mathrm{CoF}_{6}$ octahedra which share edges and corners. In this structure the cobalt and fluorine atoms are in sites with $D_{2 h}$ and $C_{2 v}$ symmetry, respectively. The Co atoms are located in the Wyckoff position (WP) $2 a(0,0,0)$ and $\mathrm{F}$ atoms are in $4 f(x, x, 0)$. Hence, the rutile structure is characterized by the lattice parameters $a$ and $c$ and the $x$ from the $4 f$ WP.

Figure 2 shows a selection of XRD patterns of $\mathrm{CoF}_{2}$ measured at different pressures up to $19.1 \mathrm{GPa}$. There is no noticeable change in the diffraction pattern up to $3.6 \mathrm{GPa}$. The Rietveld refinement for all diffraction patterns was carried out using TOPAS software. At $0.5 \mathrm{GPa}$, the lattice parameters for the tetragonal rutile $\mathrm{CoF}_{2}$ were refined to $a=4.6947$ and $c=3.1784 \AA$, which are consistent with the Inorganic Crystal Structure Database (ICSD) card No.
73460 ( $a=4.70$ and $c=3.18 \AA$ ). The lattice parameters and fractional coordinates of the rutile phase of $\mathrm{CoF}_{2}$ phase are given in Table I and are also in good agreement with those reported in previous XRD measurements, ${ }^{36} a=4.695 \AA$, $c=$ $3.182 \AA$.

All the observed peaks up to $3.6 \mathrm{GPa}$ can be indexed as rutile type. Above this pressure, some diffraction peaks progressively broaden and appear split at $6.7 \mathrm{GPa}$ as it is shown in Fig. 2. These changes in the diffraction pattern are indicative of a phase transition in $\mathrm{CoF}_{2}$ around $3.6 \mathrm{GPa}$. The new diffraction peaks in $\mathrm{CoF}_{2}$ could be assigned to the orthorhombic $\mathrm{CaCl}_{2}$-type structure (Pnnm space group). As it is observed in the volume pressure dependence in Fig. 3(a), the transition is of second order, which implies that there is not a reduction in the volume at the structural transition. According to Fig. 3(b), at the transition pressure, the lattice parameter $a$ corresponding to the rutile phase splits in $a$ and $b$ from the $\mathrm{CaCl}_{2}$-type structure, while the behavior of the $c$ parameter with respect to pressure remains almost constant. This transition has been also observed from XRD measurements and DFT calculations in other rutile fluorides and oxides such as $\mathrm{MgF}_{2},{ }^{6} \mathrm{SiO}_{2},{ }^{37}$ and $\mathrm{GeO}_{2},{ }^{38}$ to name a few. 

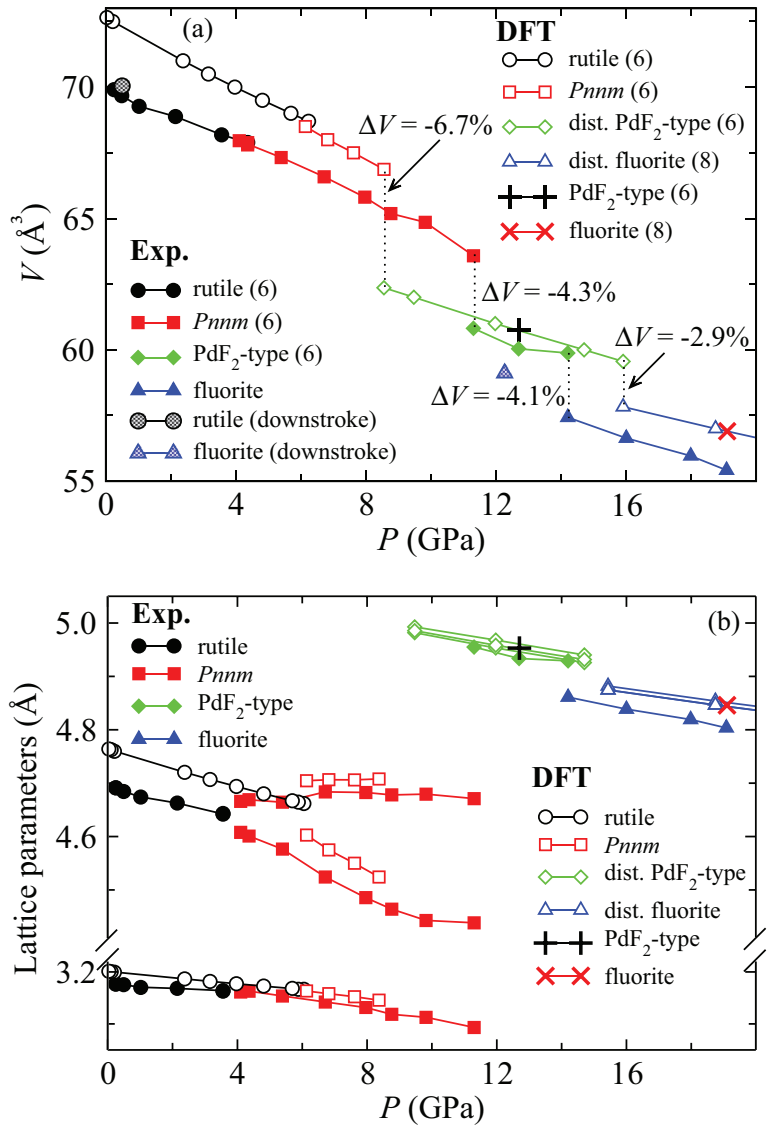

FIG. 3. (Color online) (a) Pressure dependence of the volume in the 0-20 GPa range. $\Delta V$ refers to the change in volume at the transition pressure (the minus sign indicates a contraction in volume). The coordination of $\mathrm{Co}^{2+}$ ions is depicted in parentheses (see Fig. 1). The $V(P)$ data from fluorite $(F m \overline{3} m)$ and $\mathrm{CaCl}_{2}$-type $(P n n m)$ phases have been fitted with a third-order Birch-Murnaghan EOS with a fixed value $B_{0}^{\prime}=4$. The experimental and theoretical lattice parameters for rutile and high-pressure phases of $\mathrm{CoF}_{2}$ are depicted in (b). Experimental and theoretical points are indicated with full and hollow symbols, respectively. Lines are guides for the eye.

Upon further compression in a pressure range between 10 and $14 \mathrm{GPa}$, new peaks appear in the diffraction patterns, corresponding to a phase coexistence and characterized by a crystal volume contraction of $8 \%$, within this pressure range. According to our analysis, the $\mathrm{CaCl}_{2}$-type structure and $\mathrm{PdF}_{2}$-type ( $\mathrm{Pa} \overline{3}$ space group) phases are present, as noted in Fig. 3(a). A typical Rietveld refinement of the diffraction data at $12.7 \mathrm{GPa}$ is shown in Fig. 2(a), while the lattice parameters and fractional coordinates are given in Table I. These parameters are similar to those reported in recent DFT calculations of $\mathrm{FeF}_{2}$ at $13.2 \mathrm{GPa}^{10}$ The transition $P n n m \rightarrow P a \overline{3}$ has been observed in other fluorides, such as $\mathrm{MgF}_{2}{ }^{6,8}$ However, this behavior happens to be different in the $\mathrm{CoF}_{2}$ case. According to previous work in $\mathrm{FeF}_{2}$ (Ref. 10) and $\mathrm{MnF}_{2},{ }^{39}$ this transition could be observed only under nonhydrostatic conditions, while under hydrostaticity the transition goes from $P n n m$ to a distorted $\mathrm{PdF}_{2}$-type phase with $P b c a$ space group (Fig. 1). Raman data of Fig. 3 indicate that phase coexistence of $P n n m+P a \overline{3}$ between 8 and $15 \mathrm{GPa}$ is very likely. This topic will be discussed in detail in Sec. IV E.
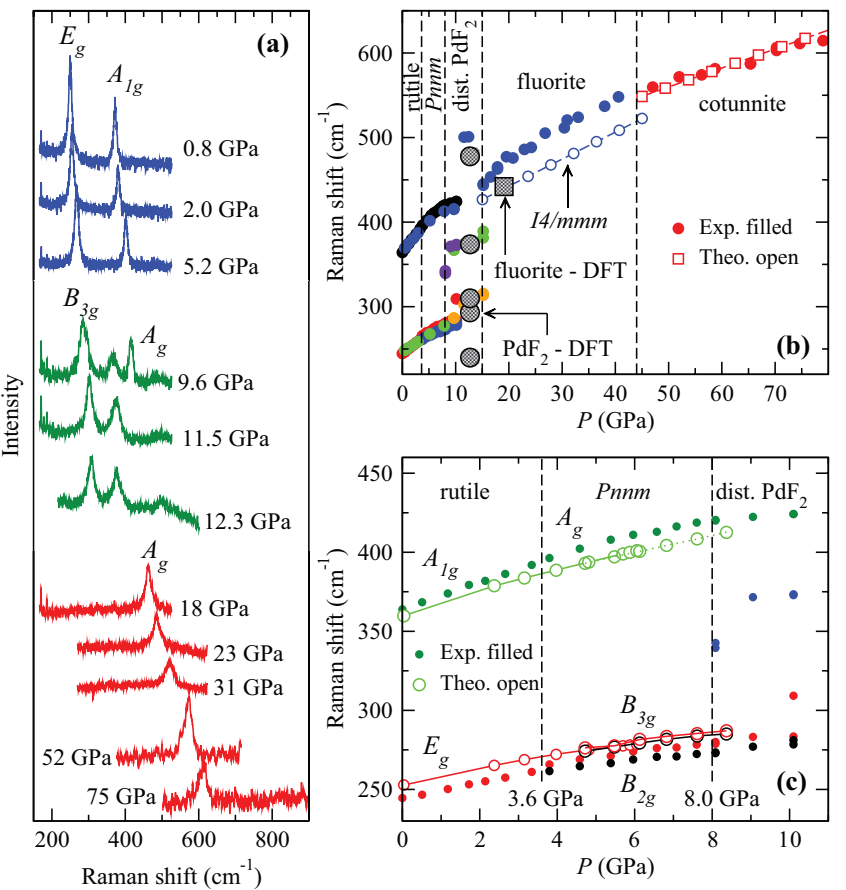

FIG. 4. (Color online) (a) Raman spectrum of single-crystal $\mathrm{CoF}_{2}$ with pressure in the $0-80 \mathrm{GPa}$ range. The corresponding Raman frequency variations are shown in (b). Note the variation of $\omega(P)$ slope and number of Raman modes at the structural phase-transition pressure. The vertical dotted lines indicate the number of phase transitions and corresponding pressure in upstroke. A magnification of $\omega(P)$ in the 0-12 GPa range is shown in (c) for detailed analysis of Raman frequencies along the rutile $\left(P 4_{2} / \mathrm{mnm}\right), \mathrm{CaCl}_{2}$-type (Pnnm), and distorted $\mathrm{PdF}_{2}$-type $(\mathrm{Pbca})$ phases. Linear fitting equations of $\omega(P)$ are given in Table II.

As pressure increases, there is another first-order phase transition from $\mathrm{CaCl}_{2}$-type structure $+\mathrm{Pa} \overline{3}$ to a fluorite phase ( $F m \overline{3} m$ space group). According to Fig. 3(a), the fluorite phase is preserved up to $19.1 \mathrm{GPa}$, which is the higher pressure reached in present XRD experiments but not in our experimental search. The lattice parameters of fluorite at this pressure are also reported in Table I. While in rutile, distorted rutile, and $P a \overline{3}$ phases, the cobalt cation $\mathrm{Co}^{2+}$ is surrounded by six fluorines, in this phase the cation $\mathrm{Co}^{2+}$ is eightfold coordinated, forming a perfect cube, as shown in Fig. 1.

The experimental pressure-volume curves shown in Fig. 3(a) were analyzed using a third-order Birch-Murnaghan equation of state (EOS). ${ }^{40}$ The bulk modulus $\left(B_{0}\right)$, its pressure derivative $\left(B_{0}^{\prime}\right)$, and the atomic volume $\left(V_{0}\right)$ at zero pressure were obtained by fitting the data from rutile and $\mathrm{CaCl}_{2}$-type structures. For both phases, $V_{0}=70.09 \AA^{3}$ and $B_{0}=104 \mathrm{GPa}$, at a fixed $B_{0}^{\prime}=4$ were obtained, whereas a value $B_{0}=102$ $\mathrm{GPa}$ is obtained if we employ $B_{0}^{\prime}=4.5$, as obtained from a first-principles calculation (Table I and Sec. IVE). The obtained bulk modulus is in good agreement with the reported value in Ref. $41\left(B_{0}=108.7 \mathrm{GPa}\right)$, thus indicating that $\mathrm{CoF}_{2}$ is the second less compressible rutile fluoride, among the magnetic $T M \mathrm{~F}_{2}$, only overtaken by $\mathrm{NiF}_{2}$. According to Refs. 10 and 41 , the values of $B_{0}$ for the rutile $T M \mathrm{~F}_{2}$ are 88, $100,108.7$ (104 in this work), and $119 \mathrm{GPa}$ for $\mathrm{MnF}_{2}, \mathrm{FeF}_{2}$, $\mathrm{CoF}_{2}$, and $\mathrm{NiF}_{2}$, respectively. 
TABLE II. Experimental (in brackets) and ab initio calculated Raman frequencies $\omega\left(\mathrm{cm}^{-1}\right)$, pressure coefficients, $\frac{\partial \omega}{\partial p}\left(\mathrm{~cm}{ }^{-1} / \mathrm{GPa}\right)$, and Grüneisen parameters $\gamma$ at the $\Gamma$ point of $\mathrm{CoF}_{2}$ in the rutile $\left(P 4_{2} / \mathrm{mnm}\right.$, at $\left.\approx 0 \mathrm{GPa}\right)$ and in the high-pressure phases $\mathrm{CaCl}{ }_{2}$ type $(P n n m$, at 7.6 GPa), distorted $\mathrm{PdF}_{2}$ type $(P b c a$, at $12 \mathrm{GPa})$, distorted fluorite $(I 4 / \mathrm{mmm}$, at $18.7 \mathrm{GPa})$, and cotunnite $(P n m a$, at $56.9 \mathrm{GPa})$. The calculated data are at $0 \mathrm{~K}$ and correspond to the phase-transition sequence under ideal hydrostatic conditions. We also have included the calculated Raman frequencies for nonhydrostatic $\mathrm{PdF}_{2}$-type and fluorite phases at the respective pressure. The calculated frequencies of infrared and silent modes together with their values of $\frac{\partial \omega}{\partial p}$ and $\gamma$ are also shown for completeness.

\begin{tabular}{|c|c|c|c|c|c|c|c|c|c|c|c|c|c|c|c|c|c|c|c|}
\hline \multicolumn{4}{|c|}{ Rutile } & \multicolumn{4}{|c|}{$\mathrm{CaCl}_{2}$ type } & \multicolumn{4}{|c|}{ dist. $\mathrm{PdF}_{2}$ type } & \multicolumn{4}{|c|}{ dist. fluorite } & \multicolumn{4}{|c|}{ Cotunnite } \\
\hline & $\omega$ & $\partial \omega / \partial p$ & $\gamma$ & & $\omega$ & $\partial \omega / \partial p$ & $\gamma$ & & $\omega$ & $\partial \omega / \partial p$ & $\gamma$ & & $\omega$ & $\partial \omega / \partial p$ & $\gamma$ & & $\omega$ & $\partial \omega / \partial p$ & $\gamma$ \\
\hline \multicolumn{20}{|c|}{ Raman modes } \\
\hline$B_{1 g}$ & 71.0 & -8.0 & -13.4 & $A_{g}$ & 95.9 & 12.8 & 14.4 & $A_{g}$ & 145.7 & 1.7 & 1.9 & $E_{g}$ & 436.9 & 3.2 & 1.5 & $A_{g}$ & 174.7 & 0.9 & 1.5 \\
\hline \multirow[t]{2}{*}{$E_{g}$} & 252.7 & 4.0 & 1.7 & $B_{1 g}$ & 256.6 & 3.3 & 1.3 & $B_{2 g}$ & 230.2 & 2.4 & 1.6 & $B_{1 g}$ & 438.9 & 3.2 & 1.5 & $B_{3 g}$ & 184.2 & 0.9 & 1.6 \\
\hline & [244.4] & {$[5.0]$} & {$[2.1]$} & $B_{2 g}$ & 283.8 & 3.1 & 1.1 & $B_{3 g}$ & 230.5 & 2.3 & 1.6 & & & & & $B_{1 g}$ & 212.8 & 0.9 & 1.3 \\
\hline \multirow[t]{2}{*}{$A_{1 g}$} & 359.8 & 6.6 & 1.9 & & [272.6] & [2.7] & {$[1.3]$} & $B_{1 g}$ & 229.1 & 2.3 & 1.6 & & Fluori & & & $A_{g}$ & 243.4 & 0.2 & 0.3 \\
\hline & [364.2] & [8.4] & {$[2.4]$} & $B_{3 g}$ & 285.4 & 3.1 & 1.1 & $A_{g}$ & 312.4 & 4.7 & 2.3 & & & & & $B_{2 g}$ & 285.7 & 0.5 & 0.5 \\
\hline \multirow[t]{18}{*}{$B_{2 g}$} & 482.4 & 7.1 & 1.6 & & [278.4] & [3.2] & {$[1.5]$} & & [308] & & & $T_{2 g}$ & 442.1 & & & $B_{2 g}$ & 296.6 & 1.1 & 1.1 \\
\hline & & & & $A_{g}$ & 408.3 & 5.4 & 1.3 & $A_{g}$ & 317.5 & 4.8 & 2.3 & & [467.5] & [3.6] & & $B_{1 g}$ & 337.4 & 1.3 & 1.2 \\
\hline & & & & & [418.0] & {$[5.4]$} & {$[1.7]$} & $B_{1 g}$ & 375.3 & 4.0 & 1.6 & & & & & $A_{g}$ & 352.0 & 1.6 & 1.4 \\
\hline & & & & $B_{1 g}$ & 532.8 & 4.3 & 0.8 & $B_{2 g}$ & 375.9 & 4.0 & 1.7 & & & & & $B_{2 g}$ & 374.1 & 1.8 & 1.5 \\
\hline & & & & & & & & $B_{3 g}$ & 376.4 & 4.1 & 1.7 & & & & & $A_{g}$ & 383.8 & 1.3 & 1.1 \\
\hline & & & & & & & & & {$[375]$} & & & & & & & $B_{3 g}$ & 432.4 & 1.6 & 1.2 \\
\hline & & & & & & & & $B_{3 g}$ & 476.5 & 4.0 & 1.3 & & & & & $A_{g}$ & 484.2 & 2.1 & 1.3 \\
\hline & & & & & & & & $B_{2 g}$ & 477.2 & 4.0 & 1.3 & & & & & $B_{2 g}$ & 481.1 & 1.5 & 1.0 \\
\hline & & & & & & & & $B_{1 g}$ & 479.3 & 4.1 & 1.3 & & & & & $B_{2 g}$ & 502.7 & 2.1 & 1.3 \\
\hline & & & & & & & & & {$[501]$} & & & & & & & $B_{3 g}$ & 524.4 & 2.5 & 1.5 \\
\hline & & & & & & & & & & & & & & & & $B_{2 g}$ & 568.8 & 2.5 & 1.4 \\
\hline & & & & & & & & & $\mathrm{PdF}_{2}$ & type & & & & & & $A_{g}$ & $\begin{array}{c}575.6 \\
{[576.2]}\end{array}$ & $\begin{array}{c}2.2 \\
{[1.8]}\end{array}$ & 1.2 \\
\hline & & & & & & & & $T_{g}$ & 239.7 & & & & & & & $B_{1 g}$ & 591.5 & 2.7 & 1.5 \\
\hline & & & & & & & & & 293.4 & & & & & & & & & & \\
\hline & & & & & & & & & 309.8 & & & & & & & & & & \\
\hline & & & & & & & & & 373.6 & & & & & & & & & & \\
\hline & & & & & & & & & 477.7 & & & & & & & & & & \\
\hline & \multicolumn{19}{|c|}{ Infrared modes } \\
\hline$E_{u}$ & 185.9 & -0.9 & -0.6 & $B_{3 u}$ & 161.8 & -3.7 & -2.2 & $B_{3 u}$ & 77.5 & -1.4 & -2.7 & $E_{u}$ & 319.8 & 3.5 & 2.3 & $B_{1 u}$ & 141.9 & 1.2 & 2.7 \\
\hline$E_{u}$ & 259.4 & 3.4 & 1.4 & $B_{2 u}$ & 192.1 & 2.1 & 1.1 & $B_{2 u}$ & 76.2 & -1.5 & -2.9 & $A_{2 u}$ & 324.2 & 3.6 & 2.3 & $B_{3 u}$ & 264.0 & 1.3 & 1.5 \\
\hline$A_{2 u}$ & 338.1 & 5.5 & 1.7 & $B_{2 u}$ & 278.3 & 1.9 & 0.7 & $B_{1 u}$ & 74.4 & -1.6 & -3.2 & & & & & $B_{1 u}$ & 326.7 & 2.1 & 1.9 \\
\hline \multirow[t]{12}{*}{$E_{u}$} & 384.1 & 9.2 & 2.5 & $B_{3 u}$ & 282.9 & 3.2 & 1.1 & $B_{2 u}$ & 216.8 & 0.6 & 0.5 & & & & & $B_{3 u}$ & 334.0 & 1.8 & 1.7 \\
\hline & & & & $B_{1 u}$ & 378.7 & 5.0 & 1.3 & $B_{3 u}$ & 216.5 & 0.6 & 0.4 & & & & & $B_{2 u}$ & 382.1 & 2.3 & 1.8 \\
\hline & & & & $B_{3 u}$ & 442.6 & 5.9 & 1.3 & $B_{1 u}$ & 218.9 & 0.7 & 0.5 & & & & & $B_{3 u}$ & 393.2 & 1.8 & 1.5 \\
\hline & & & & $B_{2 u}$ & 454.9 & 8.0 & 1.8 & $B_{2 u}$ & 309.0 & 2.6 & 1.3 & & & & & $B_{1 u}$ & 411.0 & 2.1 & 1.6 \\
\hline & & & & & & & & $B_{1 u}$ & 308.7 & 2.5 & 1.3 & & & & & $B_{3 u}$ & 451.4 & 1.7 & 1.2 \\
\hline & & & & & & & & $B_{3 u}$ & 311.1 & 2.6 & 1.3 & & & & & $B_{2 u}$ & 501.8 & 2.6 & 1.6 \\
\hline & & & & & & & & $B_{3 u}$ & 380.3 & 5.6 & 2.3 & & & & & $B_{1 u}$ & 536.8 & 2.1 & 1.2 \\
\hline & & & & & & & & $B_{1 u}$ & 385.8 & 5.7 & 2.3 & & & & & $B_{1 u}$ & 558.5 & 2.3 & 1.3 \\
\hline & & & & & & & & $B_{2 u}$ & 386.3 & 5.6 & 2.3 & & & & & $B_{3 u}$ & 597.4 & 1.8 & 1.0 \\
\hline & & & & & & & & $B_{2 u}$ & 466.3 & 5.3 & 1.8 & & & & & & & & \\
\hline & & & & & & & & $B_{1 u}$ & 465.7 & 5.4 & 1.8 & & & & & & & & \\
\hline & & & & & & & & $B_{3 u}$ & 467.4 & 5.3 & 1.8 & & & & & & & & \\
\hline \multicolumn{20}{|c|}{ Silent modes } \\
\hline$B_{1 u}$ & 155.4 & 0.4 & 0.3 & $A_{u}$ & 158.5 & 0.3 & 0.2 & $A_{u}$ & 181.8 & 0.5 & 0.4 & & & & & $A_{u}$ & 166.3 & 0.8 & 1.5 \\
\hline$A_{2 g}$ & 249.4 & 0.4 & 0.2 & $A_{u}$ & 413.8 & 5.8 & 1.4 & $A_{u}$ & 182.7 & 0.5 & 0.4 & & & & & $A_{u}$ & 382.2 & 2.4 & 2.0 \\
\hline \multirow[t]{4}{*}{$B_{1 u}$} & 363.3 & 7.6 & 2.2 & & & & & $A_{u}$ & 187.5 & 2.0 & 1.6 & & & & & $A_{u}$ & 478.3 & 2.2 & 1.4 \\
\hline & & & & & & & & $A_{u}$ & 266.9 & 0.5 & 0.3 & & & & & & & & \\
\hline & & & & & & & & $A_{u}$ & 466.1 & 5.5 & 1.8 & & & & & & & & \\
\hline & & & & & & & & $A_{u}$ & 470.4 & 5.5 & 1.8 & & & & & & & & \\
\hline
\end{tabular}


The experimental lattice parameters for rutile, Pnnm, and fluorite phases as a function of pressure are shown in Fig. 3(b). According to this figure, the $a$ lattice parameter from rutile is much more compressible than $c$, while the $b$ cell parameter from Pnnm is more compressible than the $a$ for rutile and as well as for $a$ of Pnnm. It is interesting to note that the $c$ parameter for rutile and Pnnm have the same compressibility below $10 \mathrm{GPa}$. In the fluorite phase, $a$ is less compressible than the $a$ and $b$ cell parameters from rutile and Pnnm phases, respectively.

\section{B. Raman measurements}

Figure 4(a) shows the Raman spectra of $\mathrm{CoF}_{2}$ at different pressures from 0.8 to $75 \mathrm{GPa}$. At low pressure and up to 3.6 GPa, two Raman modes have been observed for $\mathrm{CoF}_{2}$ in the rutile phase: $E_{g}$ and $A_{1 g}$. The symmetry assignment for the Raman modes has been performed in accordance with our calculations and the comparison with previous results in other rutile fluorides. ${ }^{10,42}$ Figure 4(b) shows the pressure dependence of the $\mathrm{CoF}_{2}$ Raman modes in the rutile phase and the corresponding high-pressure phases. The symmetry assignment for the Raman modes at the $\Gamma$ point along with their experimental and calculated frequencies $\omega$, pressure coefficients $\frac{\partial \omega}{\partial p}$, and their Grüneisen parameters $\gamma$ are listed in Table II. The experimental Grüneisen parameters were obtained by using the calculated $B_{0}$ values assuming $B_{0}^{\prime}=4.5$, as obtained from the theoretical results. Figure 4(c) shows the pressure evolution of the Raman modes up to $11 \mathrm{GPa}$. This figure shows that the $E_{g}$ Raman mode has a splitting at $\approx 3.6 \mathrm{GPa}$, which suggests that $\mathrm{CoF}_{2}$ undergoes a secondorder phase transition from rutile to $\mathrm{CaCl}_{2}$-type structure as determined from our XRD analysis.

The second-order phase transition observed at $3.6 \mathrm{GPa}$ (calculated at $4.7 \mathrm{GPa}$ from Raman frequencies, see Sec. IV E 2) is noteworthy. It has been reported ${ }^{10}$ that the frequency of the $B_{1 g}$ Raman mode in the rutile phase decreases with decreasing temperature or increasing pressure, in contrast to the typical behavior observed for other Raman active phonons. Thus, the Grüneisen parameter and the pressure coefficient $\partial \omega / \partial \mathrm{P}$ are negative for this mode. This softening of the $B_{1 g}$ mode is associated with the rotation of the anions around the central Co atom. A similar behavior has been observed in other isostructural rutile dioxides ${ }^{43-48}$ and difluorides ${ }^{3,7,49,50}$ such as $\mathrm{SiO}_{2}, \mathrm{RuO}_{2}, \mathrm{CrO}_{2}, \mathrm{SnO}_{2}, \mathrm{GeO}_{2}, \mathrm{MnF}_{2}$, and $\mathrm{ZnF}_{2}$. Analysis of the eigenvectors corresponding to the Raman $B_{1 g}$ softening indicates a structural instability, which is the precursor for a structural phase transition. Unfortunately, this mode in $\mathrm{CoF}_{2}$ is located at $66 \mathrm{~cm}^{-1}$ in the $10-\mathrm{K}$ Raman spectrum, ${ }^{51}$ but its intensity is too low for a suitable measurement at room temperature, even more when we are working with DAC. However, this phase transition is characterized by a splitting of the Raman active $E_{g}$ mode in the orthorhombic Pnnm phase, which according to calculations is slightly pressure dependent (see Fig. 5). Although the calculated splitting $\left(2 \mathrm{~cm}^{-1}\right)$ is below the peak width, we can follow such a splitting, and consequently determine the phase-transition pressure, by measuring the peak width of both the $E_{g}$ and $A_{1 g}$ modes as a function of pressure. Figures 5(a) and 5(b) show the variation of $E_{g}$ and $A_{1 g}$ Raman modes as a function
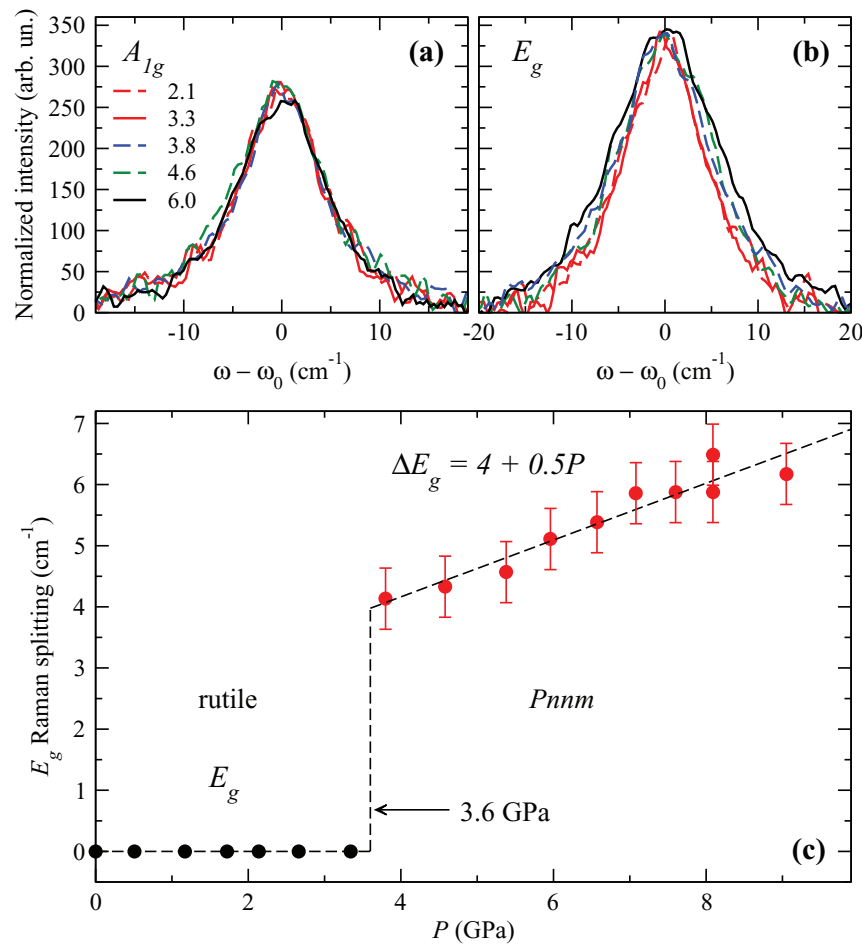

FIG. 5. (Color online) Change of the Raman active $A_{1 g}$ and $E_{g}$ peaks (rutile phase) along the rutile-to- $\mathrm{CaCl}_{2}$-type phase transition in the 0-6 GPa pressure range. The peaks exhibit a Lorentzian profile with a peak width of $9 \mathrm{~cm}^{-1}$ in both cases. Whereas the $A_{1 g}$ peak width does not vary with pressure in this range, the $E_{g}$ peak width experiences an abrupt broadening of $13 \mathrm{~cm}^{-1}$ at $3.6 \mathrm{GPa}$. In terms of $E_{g}$ splitting in the orthorhombic $\mathrm{CaCl}_{2}$-type phase, this broadening means a splitting of $4 \mathrm{~cm}^{-1}$ at $3.6 \mathrm{GPa}$, and increases linearly with pressure at a rate of $0.5 \mathrm{~cm}^{-1} / \mathrm{GPa}$.

of pressure, respectively, whereas the corresponding variation of the $E_{g}$ splitting is shown in Fig. 5(c). In the rutile phase, the Lorentzian peak width is $10 \mathrm{~cm}^{-1}$ for both $E_{g}$ and $A_{1 g}$. However, while the peak width does not change with pressure for the $A_{1 g}$ mode in the 0-6 GPa range, it behaves similarly to the $E_{g}$ mode below $3.6 \mathrm{GPa}$. Around this pressure, it abruptly broadens from 10 to $13 \mathrm{~cm}^{-1}$, thus reflecting a sudden splitting of this mode. The variation of the $E_{g}$ splitting with pressure was obtained by fitting the Raman peak to the sum of two equal-intensity Lorentzian profiles. The results of Fig. 5 indicate that the phase transition takes place at $3.6 \mathrm{GPa}$, and above this pressure the splitting increases linearly with pressure at a rate of $0.5 \mathrm{~cm}^{-1} / \mathrm{GPa}$.

A simple inspection of these figures confirms that $\mathrm{CoF}_{2}$ follows a similar structural phase-transition sequence in this pressure range as other $T M \mathrm{~F}_{2}$. Both the discontinuities in the frequency dependence with pressure and the pressure hysteresis indicate that most of them are first-order phase transitions, and take place (in upstroke) at 3.6, 8, 15, and $44 \mathrm{GPa}$. The frequency of the Raman modes, their pressure coefficients, and Grüneisen parameters are collected in Table II. With the exception of the first structural phase transition at 3.6 GPa, which is discussed in the previous section, XRD data (Fig. 2) show that the phase-transition sequence involves two 

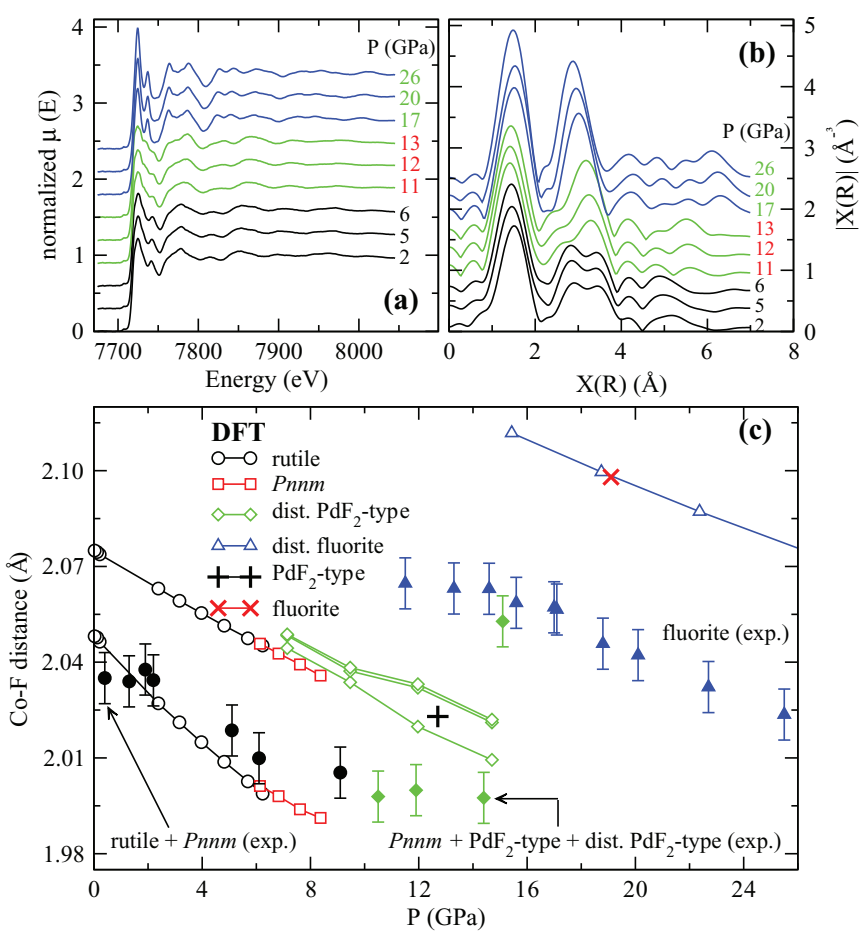

FIG. 6. (Color online) (a) Evolution of the x-ray absorption spectra of $\mathrm{CoF}_{2}$ around the $\mathrm{Co} K$ edge at $7.73 \mathrm{keV}$ with pressure. Structural phase-transition effects are clearly observed in both XANES and EXAFS regions. (b) Fourier-transform EXAFS showing the peak contributions from the first $\mathrm{Co}-\mathrm{F}$ shell and the Co-Co shells. Note that the strong backscattering from $\mathrm{Co}^{2+}$ in comparison to $\mathrm{F}^{-}$allows us to clearly detect Co-Co shell contribution. (c) Variation of the mean Co-F bond distance $R_{\mathrm{Co}-\mathrm{F}}$ with pressure. Three different regimes are observed: (1) the continuous decrease of $R_{\mathrm{Co}-\mathrm{F}}$ in the rutile and $\mathrm{CaCl}_{2-}$ type phases (sixfold coordination); (2) the stabilization of $R_{\mathrm{Co}-\mathrm{F}}$ with pressure in the $P n n m+\mathrm{PdF}_{2}$-type + dist. $\mathrm{PdF}_{2}$-type coexistence regions $(6+2$ coordination), and ( 3$)$ the abrupt increase of $R_{\mathrm{Co}-\mathrm{F}}(3 \%)$ at the phase-transition pressure to the fluorite phase (eightfold coordination). In the fluorite phase, $R_{\mathrm{Co}-\mathrm{F}}$ decreases with pressure at a rate of $3 \times 10^{-3} \AA / \mathrm{GPa}$. The experimental $R_{\mathrm{Co}-\mathrm{F}}$ values coincide with the experimental accuracy with those derived from $\mathrm{x}$-ray diffraction data. The calculated $R_{\text {Co-F }}$ values correspond to crystal phases obtained theoretically under ideal hydrostatic conditions (dist. fluorite and dist. $\mathrm{PdF}_{2}$-type) and nonhydrostatic conditions (fluorite and $\mathrm{PdF}_{2}$-type).

phases up to $15 \mathrm{GPa}$. At this pressure, the intensity pattern abruptly changes to a cubic phase (fluorite).

\section{X-ray absorption: XANES and EXAFS}

Figure 6 shows the variation of the X-ray absorption (XAS) around the Co $K$ edge, $E=7725 \mathrm{eV}$, with pressure, and the corresponding Fourier-transform $x$-ray absorption fine structure (EXAFS) $(X(R))$. A simple inspection of both X-ray absorption near-edge structure (XANES) and EXAFS regions reveals the structural phase transition between 10 to $15 \mathrm{GPa}$. The presence of peaks in $(R)$ corresponding to both the first $\mathrm{Co}-\mathrm{F}$ and $\mathrm{Co}-\mathrm{Co}$ shells is noteworthy. They provide key information about the high-pressure phases.

In the $0-15 \mathrm{GPa}$ range, $(X(R))$ shows a peak around $1.5 \AA$ which is related to the first F shell (Co-F distance), whereas the double-peak structure around $3 \AA$ is related to two different
Co-Co distance shells. This behavior is characteristic of the rutile, $\mathrm{CaCl}_{2}$-type, and orthorhombic $\mathrm{Pbca}$ structures. Above $15 \mathrm{GPa}$, the spectrum changes completely, the first peak shifts to longer Co-F distances, while the double peak transforms from a double-peak structure to a single intense Co-Co peak. This transformation confirms a structural PT to the fluoritetype structure where the $\mathrm{F}^{-}$environment of $\mathrm{Co}^{2+}$ changes from sixfold octahedral to eightfold cubic coordination. This coordination change implies an increase of the Co-F bond distance and a simplification of the $\mathrm{Co}-\mathrm{Co}$ contribution since in the fluorite phase there are 12 equivalent $\mathrm{Co}^{2+}$ next neighbors around a given $\mathrm{Co}^{2+}(F m \overline{3} m)$. The Co-F bond distance and its pressure dependence are also shown in Fig. 6(c).

\section{Optical absorption: From 6-octahedral $\mathrm{Co}^{2+}\left(d^{7}\right)$ to 8-cubic $\mathrm{Co}^{2+}\left(d^{3}\right)$}

The change of coordination around $\mathrm{Co}^{2+}$ in the pressureinduced phase transition at $15 \mathrm{GPa}$ has important consequences in the electronic properties of $\mathrm{CoF}_{2}$. In fact, such a coordination change transforms the $\mathrm{Co}^{2+}-\mathrm{F}^{-}$bonding from an octahedral local structure, where the $\sigma$-bonding interaction affects mainly

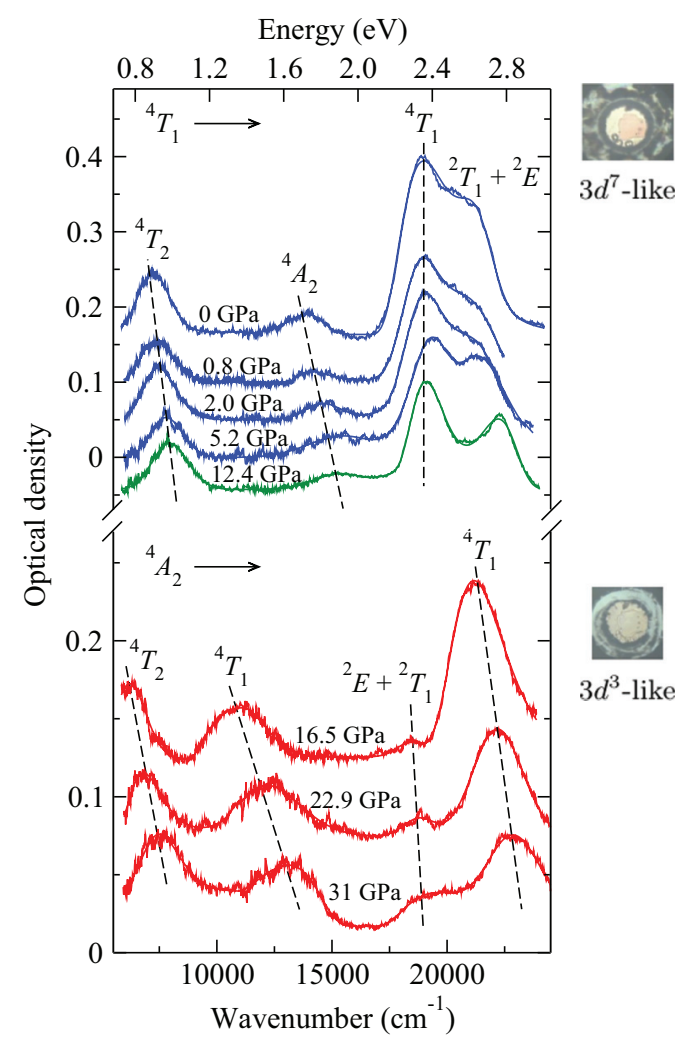

FIG. 7. (Color online) Variation of the optical absorption spectrum of $\mathrm{CoF}_{2}$ with pressure. Below $15 \mathrm{GPa}$, the spectrum consists of mainly three spin-allowed bands, which are assigned to electronic transitions between crystal-field states within $3 d^{7}$ configuration (sixfold octahedral coordination). Note that the three bands shift to higher energy with pressure according to the crystal-field increase due to $R_{\mathrm{Co}-\mathrm{F}}$ reduction. Above $15 \mathrm{GPa}$ (fluorite phase), the spectrum changes completely due to the coordination change around the $\mathrm{Co}^{2+}$ from sixfold octahedral to eightfold cubic coordination. The spectrum in this pressure range is interpreted in terms of a $3 d^{3}$ configuration (rubylike spectrum), where the first band provides directly the $t_{2 g}-e_{g}$ crystal-field splitting $\Delta$. 
the $\mathrm{Co}^{2+} e_{g}$ orbitals $\left(3 z^{2}-r^{2}, x^{2}-y^{2}\right)$ with nonbonding $t_{2 g}$ orbitals $(x z, y z, x y)$, to an eightfold coordination where the $\pi$-bonding interaction is caused by the $t_{2 g}$ orbitals. This implies a change in the electronic ground state associated with the inversion of $d$ orbitals from $\left(t_{2 g}^{5}-e_{g}^{2}\right)$ to $\left(e_{g}^{4}-t_{2 g}^{3}\right)$. The orbital triplet ${ }^{4} T_{1}$ ground state in sixfold coordination changes to the orbital singlet ${ }^{4} A_{2}$ ground state (eightfold), and consequently a change of the effective electronic configuration from $d^{7}$-electron to $d^{3}$-hole, hence the change of $d$ - $d$ electronic spectra. ${ }^{52}$ Figure 7 shows the variation of the optical absorption spectrum of $\mathrm{CoF}_{2}$ with pressure.

At ambient pressure, it consists of three main bands associated with electronic transitions from the ${ }^{4} T_{1}$ ground state to ${ }^{4} T_{2},{ }^{4} A_{2}$, and ${ }^{4} T_{1}$ excited states in order of increasing energy. In the 0-15 GPa range, pressure induces blue-shifts of the three main bands but the spectral pattern is essentially the same as the sixfold octahedral $\mathrm{CoF}_{6}$ coordination. Above $15 \mathrm{GPa}$, in the fluorite phase, the spectrum changes completely. Instead of one band around $1 \mathrm{eV}$, we observe two bands at about 0.8 and $1.2 \mathrm{eV}$. A third high-energy band is also observed at $2.5 \mathrm{eV}$. These rubylike absorption bands correspond to electronic transitions from the ${ }^{4} A_{2}$ ground state
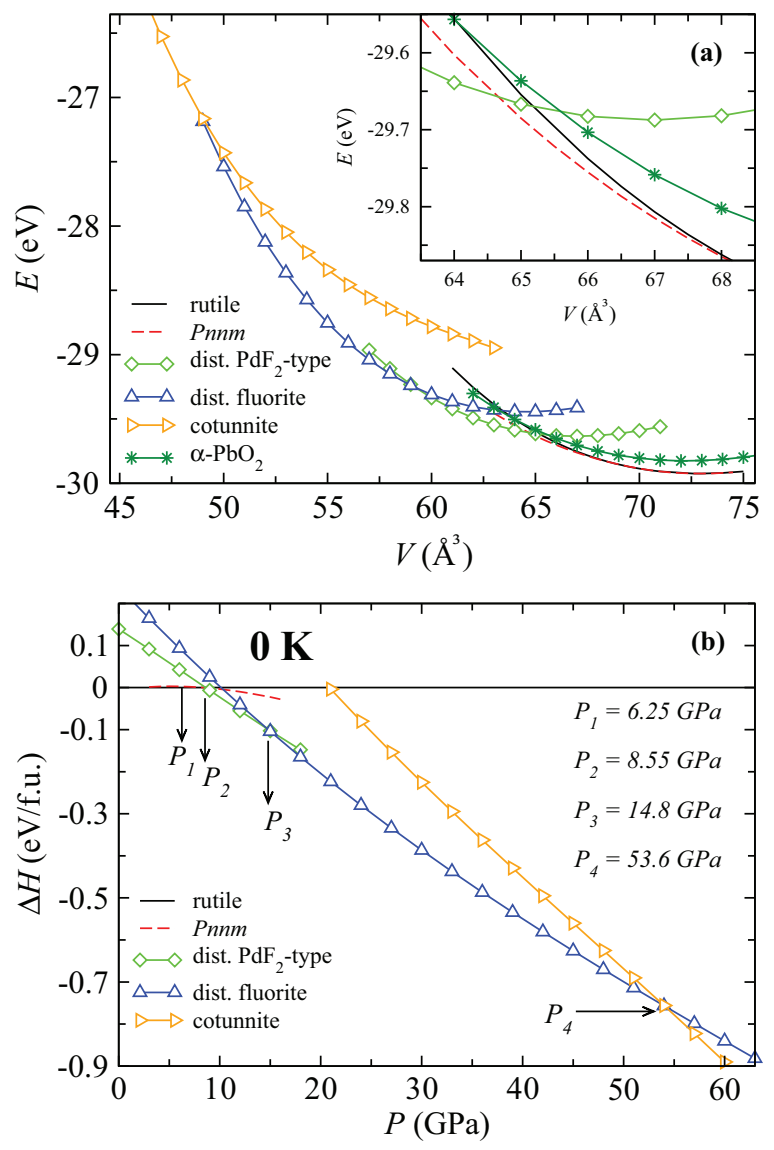

FIG. 8. (Color online) (a) Calculated total-energy-volume (per f.u.) curves for $\mathrm{CoF}_{2}$ in the rutile and in the high-pressure phases $\mathrm{CaCl}_{2}$-type $(P n n m)$, dist. $\mathrm{PdF}_{2}$-type $(P b c a)$, dist. fluorite ( $I 4 / \mathrm{mmm}$ ), cotunnite (Pnma), and $\alpha-\mathrm{PbO}_{2}$-type ( $\mathrm{Pbcn}$ ) structures. (b) Enthalpy difference as a function of pressure for $\mathrm{CoF}_{2}$ in the phases of (a). The enthalpy is measured with respect to the enthalpy of the rutile phase. to ${ }^{4} T_{2},{ }^{4} T_{1}$ (a) and ${ }^{4} T_{1}$ (b) excited states, respectively, in order of increasing energy. The first band provides directly the crystal-field splitting $\Delta$ as shown in Fig. 7, where the energy increases with pressure, i.e., decreasing the Co-F bond distance. An analysis of the $R$ dependence of $\Delta$ as $\Delta=k R^{-n}$ indicates that the exponent of such a variation in the fluorite phase is $n=5$. A more detailed account of these variations in each phase will be reported in a forthcoming paper.

\section{E. Theoretical predictions for ideal hydrostatic conditions}

\section{Structural properties}

In order to delve into the phase-transition sequence of $\mathrm{CoF}_{2}$ and some physical properties unavailable through experiments, we have explored the structural and dynamical properties under ideal hydrostatic conditions through $a b$ initio total-energy and lattice dynamics calculations. According to the literature, $\mathrm{CoF}_{2}$ is an antiferromagnetic (AFM) ionic insulator at low temperature, with a Néel temperature of $37.7 \mathrm{~K} .{ }^{53}$ In order to
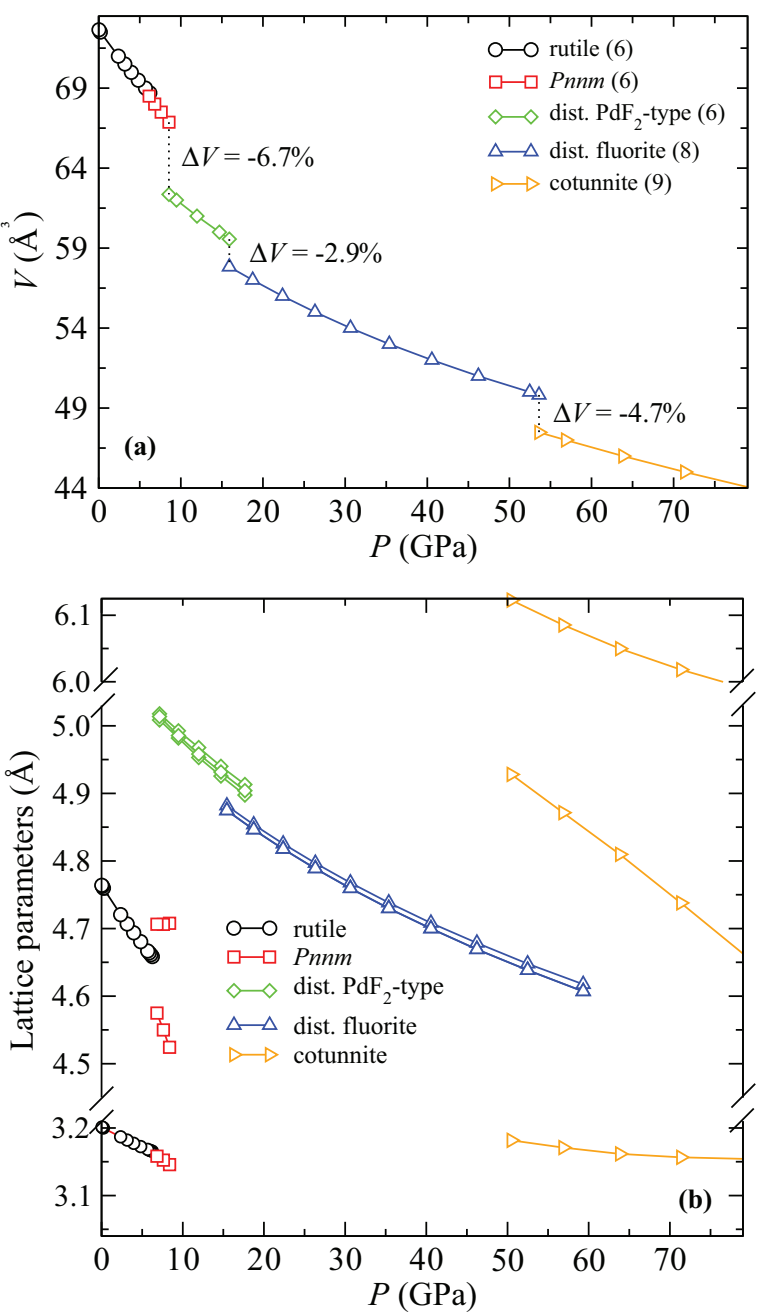

FIG. 9. (Color online) (a) Pressure dependence of the volume in the $0-80 \mathrm{GPa}$ range. $\Delta V$ refers to the change in volume at the transition pressure (the minus sign indicates a contraction in volume). The coordination of $\mathrm{Co}^{2+}$ ions is depicted in parentheses (see Fig. 1). The lattice parameters for rutile and high-pressure phases of $\mathrm{CoF}_{2}$ are depicted in (b). 
describe the proper magnetic behavior, we performed collinear calculations with the nonmagnetic (NM), AFM, and ferromagnetic (FM) spin configurations. We found that the AFM phase has the lowest energy for all the considered structures. Our computational findings show that the rutile phase has the lowest total energy, which is in good agreement with experimental results, and the crystal structure of rutile appears in Fig. 1. The equilibrium lattice parameters were calculated by minimizing the crystal total energy obtained for different volumes and fit a third-order Birch-Murnaghan EOS. ${ }^{40}$ The results for the equilibrium volume $V_{0}$, bulk modulus $B_{0}$, bulk modulus pressure derivative $B_{0}^{\prime}$, Co magnetic moment cation per f.u. $\mu_{\mathrm{Co}}$, lattice parameters $a$ and $c$, interatomic bond distance $d_{\mathrm{Co}-\mathrm{F}}$, and WPs for $\mathrm{CoF}_{2}$ are summarized in Table I. According to Table $\mathrm{I}$, the $V_{0}$ is overestimated by $\approx 3.5 \%$, as usual with the GGA approximation, which implies that $B_{0}$ is typically underestimated. ${ }^{54}$ It is also concluded from Table I that the $x$ value for $4 f$ WP is in agreement with experiments.

To study the high-pressure regime and the phase transitions driven by pressure in $\mathrm{CoF}_{2}$, we have considered several structures that were previously analyzed in the study of rutile difluorides and as well as those observed in the experimental part of this paper. The first candidate is the $\mathrm{CaCl}_{2}$-type structure, due to the observed second-order phase transition that takes the rutile to this phase. The $\mathrm{PdF}_{2}$-type one for the first-order phase transition $\mathrm{CaCl}_{2}$-type $\rightarrow \mathrm{PdF}_{2}$-type observed in $\mathrm{x}$-ray experiments as a coexistence of $\mathrm{CaCl}_{2}$-type $+\mathrm{PdF}_{2}$ type phases from 10 to $15 \mathrm{GPa}$ [see Fig. 3(a)]. The fluorite phase (here we also considered the distorted $\mathrm{PdF}_{2}$ type and the distorted fluorite to account the effects of nonhydrostaticity), and the cotunnite for the final phase transition are observed in the Raman experiments [Figs. 4(a) and 4(b)]. To broaden our structural search, other structures were also included in the study for the high-pressure phases, the $\alpha-\mathrm{PbO}_{2}$-type structure observed in other fluorides such as $\mathrm{MnF}_{2},{ }^{39}$ the badeleyite, and the distorted cubic $\mathrm{ZrO}_{2}$-type phase.

Figure 8(a) shows the energy-volume curves for the most representative polymorphs of $\mathrm{CoF}_{2}$ for which the relative stability and coexistence pressures of the phases can be extracted by the common-tangent construction. ${ }^{54}$ According to our results, the $\alpha-\mathrm{PbO}_{2}$-type structure, the badeleyite, and the $\mathrm{ZrO}_{2}$-type phase are not competitive against the proposed experimental and distorted structures. Figure 8(b) shows the evolution of enthalpy difference $\Delta H$ with pressure for rutile

TABLE III. Mechanical representation of the phonon modes for rutile $\left(P 4_{2} / m n m\right), \mathrm{CaCl}_{2}$-type structure $(P n n m)$, distorted $\mathrm{PdF} \mathrm{C}_{2}$ type $(P b c a), \mathrm{PdF}_{2}$ type $(P a \overline{3})$, distorted fluorite $(I 4 / \mathrm{mmm})$, fluorite $(F m \overline{3} \mathrm{~m})$, and cotunnite $(P n m a)$ in terms of each Wyckoff position.

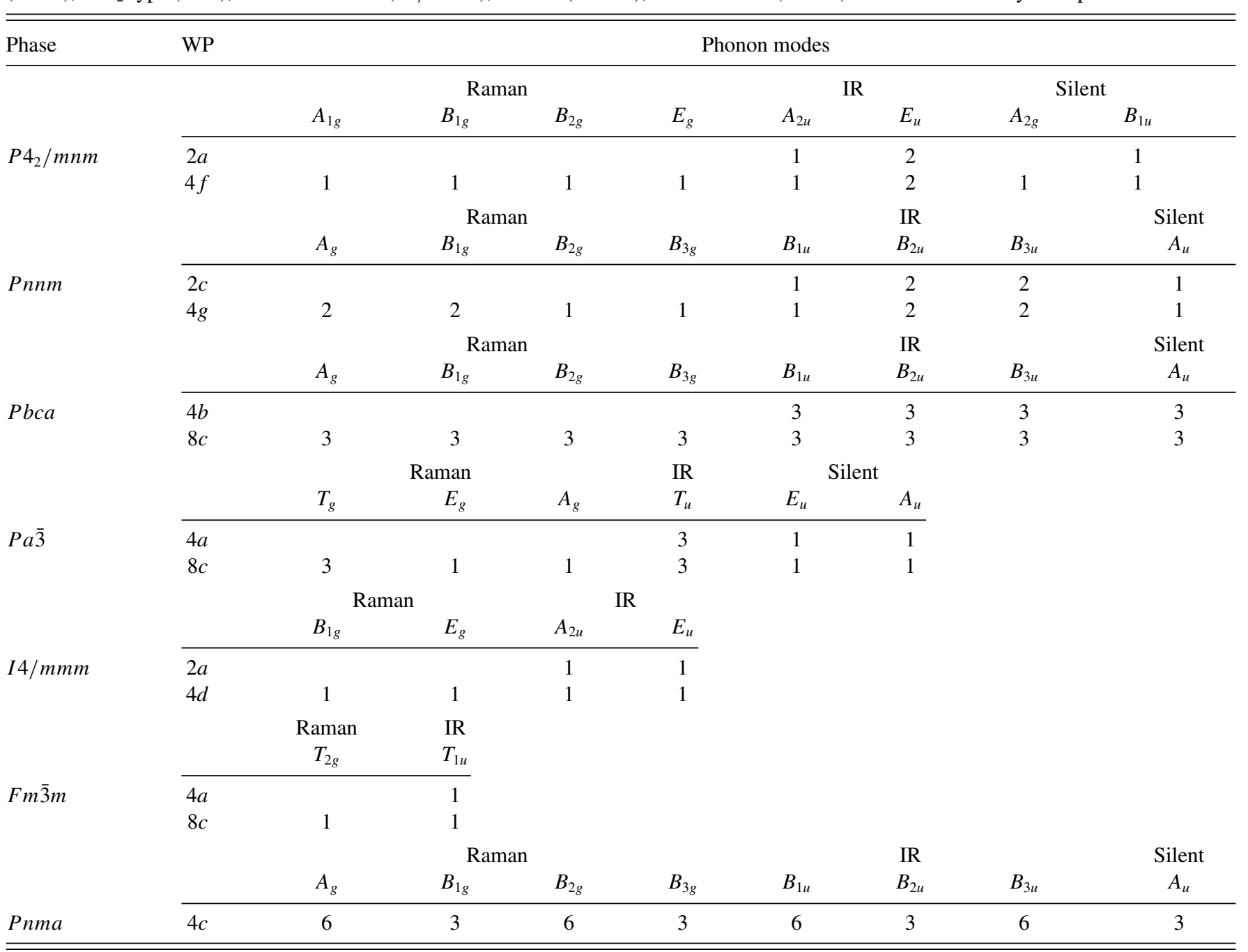


and the most relevant high-pressure phases with respect to the rutile enthalpy $H_{\text {rut }}$, where $P_{x}$ denotes the transition pressure, at each observed structural phase transition. We should stress that in traditional DFT the calculations are performed at $0 \mathrm{~K}$, so that temperature effects can be present that are not considered in this work but that can be included by using the quasiharmonic approximation, ${ }^{55}$ for example. Therefore, the pressures at which the phase transitions occur could differ a bit from those observed experimentally, as it has been reported in other compounds, where these effects are demonstrated to be relevant. ${ }^{56,57}$

According to the $\Delta H$ versus $P$ diagram, at $0 \mathrm{~K}$, we found that under hydrostatic conditions the phase transition driven by pressure can be summarized as follows: rutile $\rightarrow \mathrm{CaCl}_{2}$ type $\rightarrow$ distorted $\mathrm{PdF}_{2}$ type $\rightarrow$ distorted fluorite $\rightarrow$ cotunnite, which is similar to the transitions reported in other $T M \mathrm{~F}_{2}$ compounds such as $\mathrm{FeF}_{2}$ (Ref. 10) and $\mathrm{MnF}_{2} \cdot{ }^{39}$ Whereas if the hydrostaticity is neglected in the calculations, the path for the phase transition is rutile $\rightarrow \mathrm{CaCl}_{2}$ type $\rightarrow \mathrm{PdF}_{2}$ type $\rightarrow$ fluorite $\rightarrow$ cotunnite, as it is obtained for compounds such as $\mathrm{MgF}_{2}$ (Refs. 6 and 8) and $\mathrm{ZnF}_{2}{ }^{4}$ These results are illustrated in Fig. 1. We unveil that when nonhydrostatic conditions are considered, there is a difference of $\approx 0.3 \mathrm{GPa}$ in the stress-tensor values along the crystallographic $a, b$, and $c$ components for the $\mathrm{PdF}_{2}$-type and fluorite phases, whereas the energy difference in the enthalpy among distorted $\mathrm{PdF}_{2}$-type and $\mathrm{PdF}_{2}$-type (distorted fluorite and fluorite) phases is less than $0.3 \mathrm{meV} / \mathrm{f}$.u. This means that the lowest-energy state belongs to the phases obtained under hydrostatic conditions. Table I shows the structural parameters for phases studied under hydrostatic and nonhydrostatic conditions. Although the difference in energy is small, there are important changes between the spatial groups and some properties such as phonons. Similar results were observed for $\mathrm{FeF}_{2}$ (Ref. 10) and $\mathrm{MnF}_{2} \cdot{ }^{39}$ For the sake of simplicity, in what follows we limit our discussions to phases obtained under hydrostatic conditions.

According to Fig. 8(b), as pressure increases to $\approx 6.25 \mathrm{GPa}$, there is a phase transition from rutile to $\mathrm{CaCl}_{2}$-type structure. This transition is also observed in the experimental pressure dependence of volume and lattice parameters from Figs. 9(a) and 9(b), respectively. According to Fig. 6(c), passing the transition pressure, there are only small changes in the interatomic bond distances $d_{\mathrm{Co}-\mathrm{F}}$ as $\mathrm{CaCl}_{2}$-type structure is obtained basically from almost rigid rotations of the $\mathrm{CoF}_{6}$ polyhedra around the $c$ axis. Upon further compression, there is a first-order phase transition to the distorted $\mathrm{PdF}_{2}$-type phase at $\approx 8.55 \mathrm{GPa}$. This transition involves a $\Delta V$ contraction of $6.7 \%$. In this phase, we observe a very small difference between the corresponding lattice parameters, less than 0.02 $\AA$ [see Table I and Fig. 9(b)], which is within the experimental error bars. In the range of stability of this phase it is observed that the interatomic bond distances $d_{\mathrm{Co}-\mathrm{F}}$ get larger as pressure increases, as can be seen in Fig. 6(b). This phase is stable up to $\approx 14.8 \mathrm{GPa}$ where the second first-order phase transition occurs from a distorted $\mathrm{PdF}_{2}$-type phase to a distorted fluorite. Here, there is a volume change of $\approx 2.9 \%$. The distorted fluorite phase is a tetragonal structure with two f.u. An important fact in this transition is the change in the cobalt atomic coordination from 6 to 8 , from a distorted polyhedra to a perfect cube, as it was discussed previously (see Fig. 1). For instructive purposes, it is better to observe the distortion of the cubic phase by plotting the lattice parameters for the distorted fluorite phase which has 4 f.u. instead of the 2 f.u. from the tetragonal $I 4 / \mathrm{mmm}$ phase as shown in Fig. 9(b) (i.e., $a$ from $I 4 / \mathrm{mmm}$ is half of $a$ parameter from the distorted fluorite). It is clear that the difference in the lattice parameters increases gradually with pressure. However, this distortion does not affect the interatomic bond distance as observed in Fig. 6(c). We found that this phase is stable in a very large pressure range from $\approx 15.9$ to $\approx 53.6 \mathrm{GPa}$. After that, there is a first-order phase transition to the cotunnite phase with a volume change of $\Delta V=4.7 \%$. This transition involves a change in coordination from 8 to 9 , where the polyhedron in this phase is very irregular with five different interatomic bond distances as indicated in Table I and Fig. 1. According to Fig. 9(b), this phase has almost the same compressibility as the distorted fluorite. However, the lattice parameter $a$ is much more compressible than the other two lattice parameters. This is due to the fact that the longest $d_{\text {Co-F }}$ is located mostly along this crystallographic direction. The $\mathrm{CoF}_{2}$ compound is stable in the cotunnite phase up to 80 $\mathrm{GPa}$, which is the largest pressure reached in this study.

\section{Vibrational properties}

Table III shows the mechanical representation of the phonon modes for the rutile and the high-pressure phases, where the corresponding modes for each Wyckoff position are clearly depicted. $A$ and $B$ modes are nondegenerate,
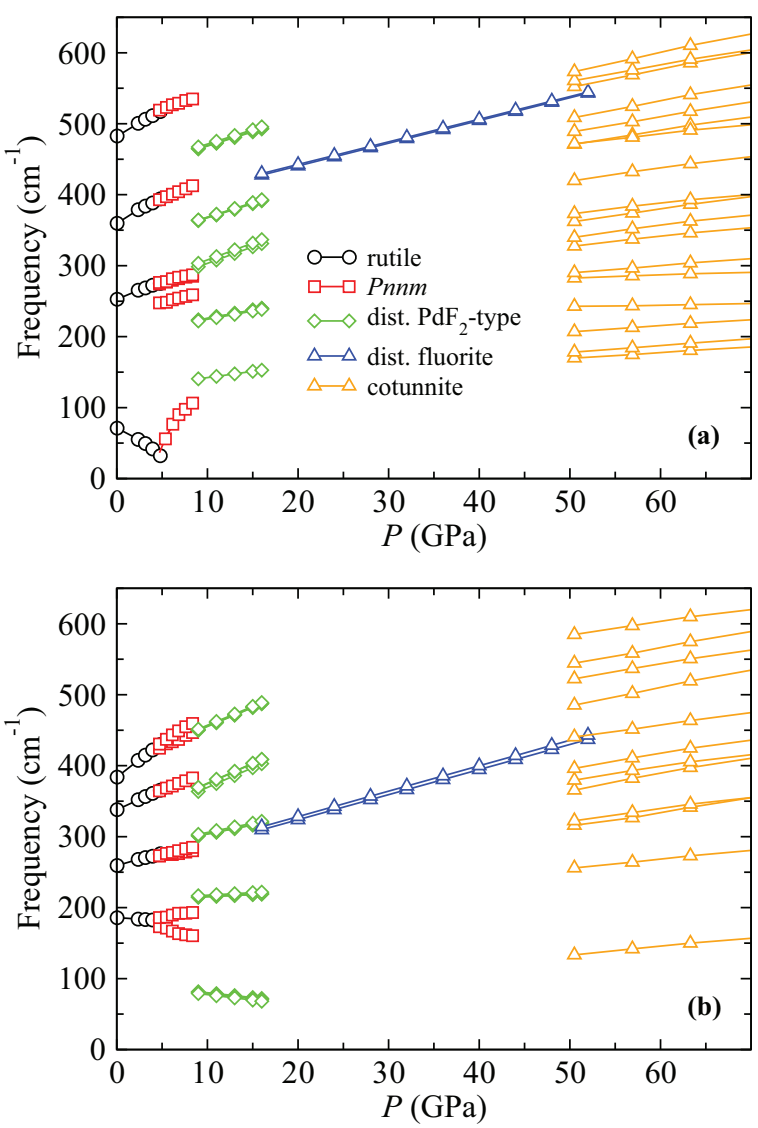

FIG. 10. (Color online) Pressure dependence of calculated (a) Raman and (b) IR mode frequencies of rutile and high-pressure phases. 
whereas the $E$ modes are doubly degenerate. At the $\Gamma$ point, each phase has three zero-frequency acoustic modes: for rutile and distorted fluorite they are $A_{2 u}+E_{u}$, whereas for orthorhombic phases $\mathrm{CaCl}_{2}$ type, distorted $\mathrm{PdF}_{2}$ type, and cotunnite they are $B_{1 u}+B_{2 u}+B_{3 u}$. The phonon frequencies $\omega$, pressure coefficients $\frac{\partial \omega}{\partial p}$, and Grüneisen parameters $\gamma$ for rutile and high-pressure phases are listed in Table II. Additionally, in Figs. 4(b) and 4(c), the calculated Raman frequencies are plotted against pressure, with fair comparison with experimental measurements, which are also reported.

Cobalt (II) fluoride, as other rutile compounds, presents a second-order phase transition driven by pressure, from rutile to $\mathrm{CaCl}_{2}$-type structure. The nature of this transition implies a phonon mode correlation between the rutile and $\mathrm{CaCl}_{2}$-type structures. As already mentioned, at the transition pressure, some modes from the rutile phase split in other phonon modes in the $\mathrm{CaCl}_{2}$-type structure: the $E_{g}$ splits into the $B_{2 g}+B_{3 g}$, and the $E_{u}$ into the $B_{2 u}+B_{3 u} .{ }^{10}$ These conclusions are well illustrated in Fig. 10, where it is shown the pressure dependence of the calculated (a) Raman and (b) IR mode frequencies at the $\Gamma$ point from the rutile and high-pressure phases. According to this, the transition rutile $\rightarrow \mathrm{CaCl}_{2}$-type structure occurs at $\approx 4.7 \mathrm{GPa}$. This transition pressure is smaller than that obtained from the $\Delta H-P$ diagram of Fig. 8(b). This difference could be due to temperature effects not considered in our calculations.

As it was pointed out in the structural properties section, the $P b c a$ phase is a distortion of a cubic phase called $\mathrm{PdF}_{2}$ type. As a result, the Raman $\left(3 T_{g}\right.$ and $\left.E_{g}\right)$ and IR $\left(6 T_{u}\right)$ phonon modes of the $\mathrm{PdF}_{2}$-type phase split into the Raman $\left(3 B_{1 g}+3 B_{2 g}+3 B_{3 g}\right.$ and $\left.2 A_{g}\right)$ and IR $\left(6 B_{1 u}+6 B_{2 u}+6 B_{3 u}\right)$ phonon modes of the distorted $\mathrm{PdF}_{2}$ type, respectively. Therefore, the difference among the Raman frequencies $\left(B_{1 g} B_{2 g}+B_{3 g}\right.$, and the $\left.A_{g}\right)$ and IR $\left(B_{1 u}+B_{2 u}+B_{3}\right)$ modes for the corresponding $T_{g}, E_{g}$, and $T_{u}$ modes are small mainly due to the slight cell-structure distortion (see Table II). Note that in the distorted $\mathrm{PdF}_{2}$-type phase, three IR modes $\left(B_{1 u}+B_{2 u}+B_{3 u}\right)$ are acoustic at the $\Gamma$ point. Table II shows that in this phase there are three IR phonon modes with negative pressure coefficient with very similar values. This behavior is due to the fact that these modes correspond to one $T_{u}$ mode from the $\mathrm{PdF}_{2}$-type phase.

The distorted fluorite phase behaves somewhat similar to the distorted $\mathrm{PdF}_{2}$ type. Here, we have 2 Raman $\left(B_{1 g}+E_{g}\right)$ and 4 IR $\left(2 A_{2 u}+2 E_{u}\right)$ phonon modes which result from the split of the $T_{2 g}+2 T_{1 u}$ phonon modes from the fluorite phase, where one $A_{2 u}$ and one $E_{u}$ correspond to the zero-frequency acoustic phonon modes at the $\Gamma$ point. In this phase, there are important differences in the phonon frequencies between experimental and theoretical results (Table II). However, both the theoretical and experimental pressure coefficients have similar values.

In the cotunnite phase, there is no negative pressure coefficient. Here, we found a very good agreement between the experimental and theoretical results for the $A_{g}$ Raman mode. Generally, we observe that the pressure coefficient for rutile-type compounds in the cotunnite phase follows the trend $\left(\frac{\partial \omega}{\partial P}\right)_{\mathrm{Co}}<\left(\frac{\partial \omega}{\partial P}\right)_{\mathrm{Fe}}<\left(\frac{\partial \omega}{\partial P}\right)_{\mathrm{Mn}}$, for which is reported a cation ionic radii of $0.90,0.92$, and $0.96 \AA$ (Ref. 58) for eightfold
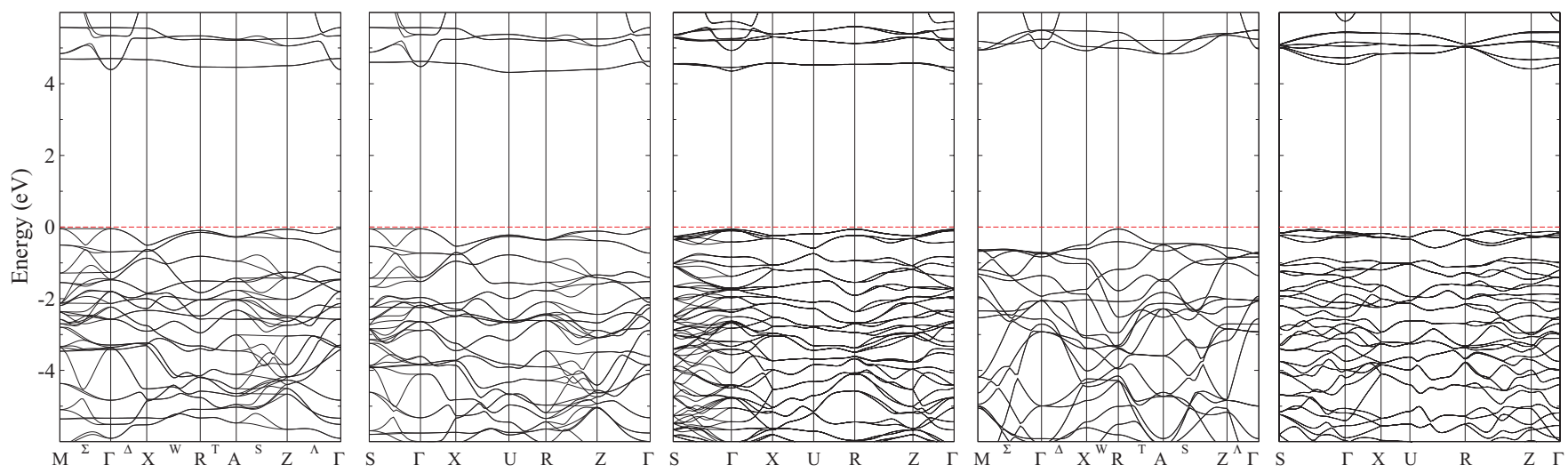

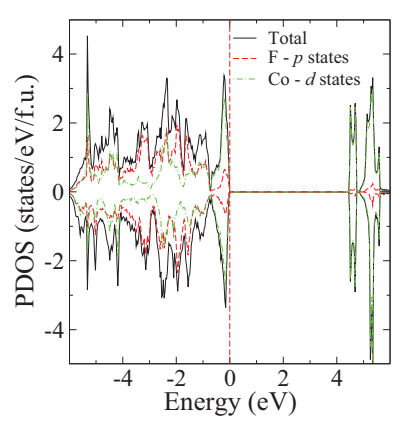

(a) rutile

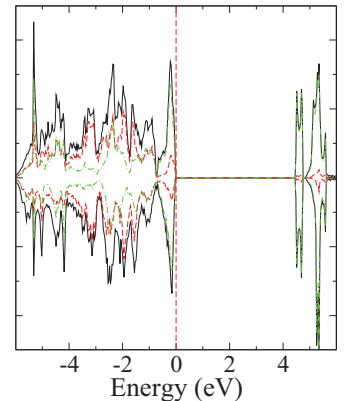

(b) $\mathrm{CaCl}_{2}$-type

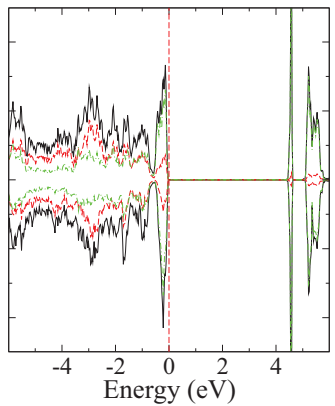

(c) dist. $\mathrm{PdF}_{2}$-type

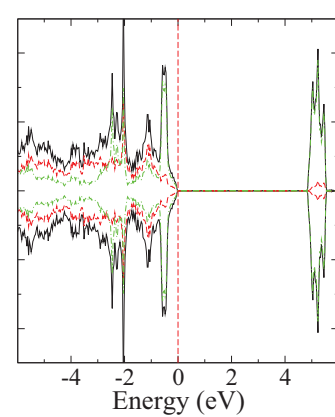

(d) dist. fluorite

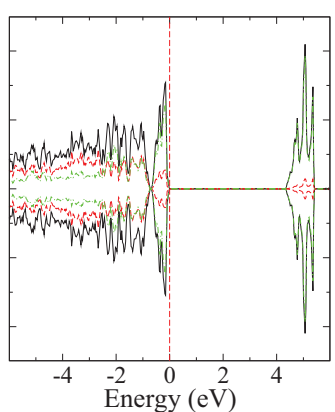

(e) cotunnite

FIG. 11. (Color online) Top row: Electronic band structure of $\mathrm{CoF}_{2}$ of rutile and high-pressure phases along the high-symmetry points of the respective Brillouin zone from Fig. 11. Bottom row: Electronic density of states (DOS) of each phase. The red dotted line at $0 \mathrm{eV}$ marks the Fermi level. 


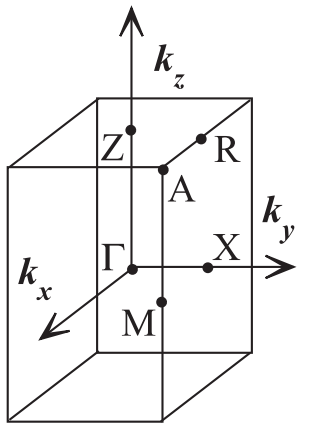

(a)

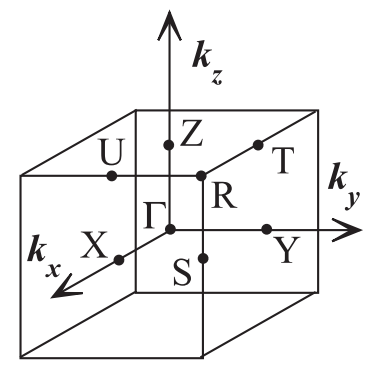

(b)
FIG. 12. Brillouin zones for (a) rutile and dist. fluorite, and (b) $\mathrm{CaCl}_{2}$-type, dist. $\mathrm{PdF}_{2}$-type, and cotunnite phases.

coordination (i.e., as ionic radius of the $T M \mathrm{~F}_{2}$ increases so does the pressure coefficient in the cotunnite phase).

\section{Electronic properties}

The electronic band structures and the density of states (DOS) for the rutile and high-pressure phases are reported in Fig. 11, whereas the Brillouin zone (BZ) for each crystal phase appears in Fig. 12. The density of states shows quite clearly that all phases are antiferromagnetic with a perfect imaging between populated majority and minority bands. The electronic energy gap and its pressure dependence are reported in Table IV. In general terms, the insulator characteristic of $\mathrm{CoF}_{2}$ remains as a function of pressure and the value of the electronic gap is barely affected and even remains the indirect character starting from the rutile phase to high-pressure phases. The only main feature to highlight is basically that the gap changes from $M-\Gamma$ to another vector in the Brillouin zone. The largest change occurs for the $I 4 / \mathrm{mmm}$ structure, where the gap is $4.88 \mathrm{eV}$ compared to $4.44 \mathrm{eV}$ at the rutile phase, but with very similar pressure dependence. The band dispersion close to the Fermi level is small, indicating a large localization, mostly from cobalt $d$ states, as observed from Fig. 11. This feature is also observed in the conduction bands, where the lowest bands are weakly dispersive and also come from cobalt $d$ states. The only difference observed is again in the case of $I 4 / \mathrm{mmm}$ structure, where there is an important shift of the band structure, the electron population close to the Fermi level is largely depleted, and the main peak observed at the Fermi level for the other phases is shifted down by a bit more than
$0.1 \mathrm{eV}$. There is a single contribution to the conduction bands from the parabolic band.

\section{CONCLUSIONS}

We have demonstrated that $\mathrm{CoF}_{2}$ experiences a structural phase-transition sequence with pressure similar to other $T M \mathrm{~F}_{2}$ like $\mathrm{FeF}_{2}$. DFT calculations are in fair agreement with structural phase-transition sequence observed in the Raman and XRD results. Moreover, a phase coexistence is observed and the observed stabilization of the fluorite-type phase under nonhydrostatic conditions is clarified through our theoretical calculations. The observed phase-transition sequence under nonhydrostatic conditions at room temperature, according to XRD and Raman experiments (Fig. 4) is rutile $\rightarrow 3.6$ $\mathrm{GPa} \rightarrow \mathrm{CaCl}_{2}$-type phase $\rightarrow 8 \mathrm{GPa} \rightarrow$ phase coexistence $(P n n m+P a \overline{3}+P b c a) \rightarrow 15 \mathrm{GPa} \rightarrow$ fluorite $\rightarrow 44 \mathrm{GPa} \rightarrow$ cotunnite. The calculated sequence under ideal hydrostatic condition at $0 \mathrm{~K}$ is rutile $\rightarrow 6.25 \mathrm{GPa} \rightarrow \mathrm{CaCl}_{2}$-type phase $\rightarrow$ $8.55 \mathrm{GPa} \rightarrow$ distorted $\mathrm{PdF}_{2}$ type $\rightarrow 14.8 \mathrm{GPa} \rightarrow$ distorted fluorite $\rightarrow 53.6 \mathrm{GPa} \rightarrow$ cotunnite. The first rutile to $\mathrm{CaCl}_{2}$-type phase transition happens to be of second order as it does not involve volume change. However, it splits the $E_{g}$ Raman mode, which was detected by a peak broadening at the phasetransition pressure. A salient feature is the characterization of a structural phase transition to the fluorite-type structure at $15 \mathrm{GPa}$, involving a drastic change of coordination from sixfold octahedral to eightfold cubic. The calculated total volume collapse between 10 and $15 \mathrm{GPa}$ is about $9 \%$ close to the measured experimental value. Under these conditions, the tetragonal distorted fluorite phase is stabilized instead of the cubic fluorite structure observed experimentally. Nevertheless, consideration of nonhydrostaticity in the calculations reveals that the distorted fluorite phase is not stable and the cubic fluorite is eventually stabilized. This surprising result explains why the pioneering works of $\mathrm{MnF}_{2}$ and $\mathrm{CoF}_{2}$ have reported that the fluorite-type phase corresponds to the high-pressure phase for both materials, despite that such a cubic structure could not be stabilized under hydrostatic conditions. Simulations also reveal that instead of the orthorhombic Pbca structure, the cubic $\mathrm{PdF}_{2}$ type is stabilized prior to the fluorite phase under nonhydrostatic conditions. However, we experimentally detect traces of both the distorted $\mathrm{PdF}_{2}$-type and the $\mathrm{PdF}_{2}$-type phases, thus suggesting a coexistence of these two phases, making the analysis of XRD data difficult. The fact that

TABLE IV. Calculated direct-energy gap among the special $k$ points of the BZ zone, energy gap $\Delta_{g}$, and the pressure variation of the $\Delta_{g}$ of $\mathrm{CoF}_{2}$ for rutile and the high-pressure phases at the specified pressure.

\begin{tabular}{|c|c|c|c|c|c|c|c|c|c|}
\hline $\begin{array}{l}\text { Tetragonal } \\
\text { phases }\end{array}$ & $\begin{array}{c}P \\
(\mathrm{GPa})\end{array}$ & $\begin{array}{c}M-M \\
(\mathrm{eV})\end{array}$ & $\begin{array}{l}\Gamma-\Gamma \\
(\mathrm{eV})\end{array}$ & $\begin{array}{l}X-X \\
(e V)\end{array}$ & $\begin{array}{l}R-R \\
(\mathrm{eV})\end{array}$ & $\begin{array}{l}A-A \\
(\mathrm{eV})\end{array}$ & $\begin{array}{l}Z-Z \\
(\mathrm{eV})\end{array}$ & $\begin{array}{c}\Delta_{g} \\
(\mathrm{eV})\end{array}$ & $\begin{array}{c}\partial \Delta_{g} / \partial p \\
(\mathrm{meV} / \mathrm{GPa})\end{array}$ \\
\hline Rutile & $\approx 0$ & 4.76 & 4.45 & 5.14 & 4.97 & 4.71 & 4.56 & $4.44(M-\Gamma)$ & 2 \\
\hline dist. fluorite & 19.1 & 5.58 & 5.69 & 5.39 & 5.22 & 5.29 & 5.92 & $4.88(R-A)$ & 2 \\
\hline $\begin{array}{l}\text { Orthorhombic } \\
\text { phases }\end{array}$ & $\begin{array}{c}P \\
(\mathrm{GPa})\end{array}$ & $\begin{array}{l}S-S \\
(\mathrm{eV})\end{array}$ & $\begin{array}{l}\Gamma-\Gamma \\
(\mathrm{eV})\end{array}$ & $\begin{array}{l}X-X \\
(\mathrm{eV})\end{array}$ & $\begin{array}{l}U-U \\
(\mathrm{eV})\end{array}$ & $\begin{array}{l}R-R \\
(\mathrm{eV})\end{array}$ & $\begin{array}{l}Z-Z \\
(\mathrm{eV})\end{array}$ & $\begin{array}{c}\Delta_{g} \\
(\mathrm{eV})\end{array}$ & $\begin{array}{c}\partial \Delta_{g} / \partial p \\
(\mathrm{meV} / \mathrm{GPa})\end{array}$ \\
\hline $\mathrm{CaCl}_{2}$ type & 8.0 & 4.66 & 4.53 & 5.17 & 4.63 & 4.73 & 4.52 & $4.37(\Gamma-U)$ & -22 \\
\hline dist. $\mathrm{PdF}_{2}$ type & 12.7 & 4.84 & 4.42 & 4.81 & 4.70 & 5.18 & 5.55 & $4.41(R-\Gamma)$ & 1 \\
\hline Cotunnite & 56.8 & 5.17 & 4.68 & 4.99 & 5.12 & 5.27 & 4.60 & $4.47(S-\Gamma-\Gamma)$ & 7 \\
\hline
\end{tabular}


the energy difference in the enthalpy among $P b c a$ and $P a \overline{3}$ phases is less than $0.3 \mathrm{meV} /$ f.u. justifies the observed puzzling behavior of $\mathrm{CoF}_{2}$ in the $10-15 \mathrm{GPa}$ range. Regarding the phase-transition pressures, the differences observed between theoretical and experimental data could be due to temperature effects since our calculations were done at $0 \mathrm{~K}$, then we only considered the enthalpy versus pressure phase diagram instead of the Gibbs versus pressure which could be done at temperatures $\neq 0 \mathrm{~K}$.

The influence of the transition-metal ion in the phasetransition sequence under pressure in $T M \mathrm{~F}_{2}$ is noteworthy. We conclude that more than the ionic radii (stereochemistry basis), $d$ bonding plays an important role in the stabilization of given phases at high pressure. The similitude between the phase-transition sequence exhibited by $\mathrm{CoF}_{2}\left(3 d^{7}\right)$ and $\mathrm{FeF}_{2}\left(3 d^{6}\right)$ in comparison to $\mathrm{ZnF}_{2}$ and $\mathrm{MgF}_{2}$ (closed-shell configurations) underlines the strong influence of an open $d$-orbital configuration $\left(3 d^{7}\right.$ or $\left.3 d^{6}\right)$ in stabilizing different phases upon compression. The Raman mode frequency and its pressure dependence are close to those obtained from first-principles calculations. This point is important since many of the vibrational modes are neither IR nor Raman active or even may be silent in pressure experiments, and therefore the calculated data as compared to the experimental data allow us to get a full description of the vibrational and elastic properties of the $\mathrm{CoF}_{2}$ following the proposed first-principles DFT methodology. We also note that the electronic properties are weakly sensitive to pressure even to values of $80 \mathrm{GPa}$.

\section{ACKNOWLEDGMENTS}

Financial support from the Spanish Ministerio de Economia y Competitividad (Projects No. MAT2010-21270-C04-03 and No. MAT2012-38664-C02-1) and MALTA INGENIOCONSOLIDER 2010 (Ref. No. CDS2007-0045) and a Technical Grant (Ref. No. PTA2011-5461-I) is acknowledged. XRD experiments were performed at MSPD beamline at ALBA Synchrotron Light Facility with the collaboration of ALBA staff (Ref. No. ID2012010239). XAS experiments were performed at ODE beamline at SOLEIL Synchrotron Light Facility (Ref. No. 20090666). We thank J. Baruchel for the loan of $\mathrm{CoF}_{2}$ single crystals. We also acknowledge the computing time provided by Red Española de Supercomputación (RES), TACC-Texas Supercomputer Center, and MALTA-Cluster.
*Corresponding author: 1sinhue@yahoo.com.mx

${ }^{1}$ L.-c. Ming and M. H. Manghnani, Geophys. Res. Lett. 5, 491 (1978).

${ }^{2}$ L. Ming, M. Manghnani, T. Matsui, and J. Jamieson, Phys. Earth Planet. Inter. 23, 276 (1980).

${ }^{3}$ J. Haines, J. M. Léger, and S. Hoyau, J. Phys. Chem. Solids 56, 965 (1995).

${ }^{4}$ X. Wu, S. Qin, and Z. Wu, Phys. Rev. B 73, 134103 (2006).

${ }^{5}$ J. D. Jorgensen, T. G. Worlton, and J. C. Jamieson, Phys. Rev. B 17, 2212 (1978).

${ }^{6}$ J. Haines, J. M. Léger, F. Gorelli, D. D. Klug, J. S. Tse, and Z. Q. Li, Phys. Rev. B 64, 134110 (2001).

${ }^{7}$ A. Perakis, D. Lampakis, Y. C. Boulmetis, and C. Raptis, Phys. Rev. B 72, 144108 (2005).

${ }^{8}$ L. Zhang, Y. Wang, T. Cui, Y. Ma, and G. Zou, Solid State Commun. 145, 283 (2008).

${ }^{9} \mathrm{X}$. Wu and Z. Wu, Eur. Phys. J. B 50, 521 (2006).

${ }^{10}$ S. López-Moreno, A. H. Romero, J. Mejía-López, A. Muñoz, and I. V. Roshchin, Phys. Rev. B 85, 134110 (2012).

${ }^{11}$ J. M. Léger, J. Haines, and C. Danneels, J. Phys. Chem. Solids 59, 1199 (1998).

${ }^{12}$ M. Wevers, J. Schn, and M. Jansen, J. Solid State Chem. 136, 233 (1998).

${ }^{13}$ C. Vettier and W. Yelon, J. Phys. Chem. Solids 36, 401 (1975).

${ }^{14}$ G. K. Rozenberg, M. P. Pasternak, P. Gorodetsky, W. M. Xu, L. S. Dubrovinsky, T. Le Bihan, and R. D. Taylor, Phys. Rev. B 79, 214105 (2009).

${ }^{15}$ A. R. Oganov, G. D. Price, and S. Scandolo, Z. Kristallogr. 220, 531 (2005).

${ }^{16}$ D. Andrault, G. Fiquet, F. Guyot, and M. Hanfland, Science 282, 720 (1998).

${ }^{17}$ V. Prakapenka, G. Shen, L. Dubrovinsky, M. Rivers, and S. Sutton, J. Phys. Chem. Solids 65, 1537 (2004).

${ }^{18}$ R. D. Shannon, Acta Crystallogr., Sect. A 32, 751 (1976).

${ }^{19}$ P. I. Sorantin and K. Schwarz, Inorg. Chem. 31, 567 (1992).
${ }^{20}$ J. A. Barreda-Argüeso and F. Rodríguez (unpublished).

${ }^{21}$ F. Baudelet, Q. Kong, L. Nataf, J. D. Cafun, A. Congeduti, A. Monza, S. Chagnot, and J. P. Itié, High Press. Res. 31, 136 (2011).

${ }^{22}$ R. A. Forman, G. J. Piermarini, J. D. Barnett, and S. Block, Science 176, 284 (1972).

${ }^{23}$ K. Syassen, High Pressure Res. 28, 75 (2008).

${ }^{24}$ K. Momma and F. Izumi, J. Appl. Crystallogr. 44, 1272 (2011).

${ }^{25}$ P. E. Blöchl, Phys. Rev. B 50, 17953 (1994).

${ }^{26}$ G. Kresse and D. Joubert, Phys. Rev. B 59, 1758 (1999).

${ }^{27}$ G. Kresse and J. Hafner, Phys. Rev. B 47, 558 (1993).

${ }^{28}$ G. Kresse and J. Hafner, Phys. Rev. B 49, 14251 (1994).

${ }^{29}$ G. Kresse and J. Furthmüller, Comput. Mater. Sci. 6, 15 (1996).

${ }^{30}$ G. Kresse and J. Furthmüller, Phys. Rev. B 54, 11169 (1996).

${ }^{31}$ J. P. Perdew, K. Burke, and M. Ernzerhof, Phys. Rev. Lett. 77, 3865 (1996).

${ }^{32}$ S. L. Dudarev, G. A. Botton, S. Y. Savrasov, C. J. Humphreys, and A. P. Sutton, Phys. Rev. B 57, 1505 (1998).

${ }^{33}$ J. Ruiz-Fuertes, D. Errandonea, S. López-Moreno, J. González, O. Gomis, R. Vilaplana, F. J. Manjón, A. Muñoz, P. RodriiguezHernández, A. Friedrich, I. A. Tupitsyna, and L. L. Nagornaya, Phys. Rev. B 83, 214112 (2011).

${ }^{34}$ H. J. Monkhorst and J. D. Pack, Phys. Rev. B 13, 5188 (1976).

${ }^{35}$ K. Parlinski, computer code PHONON. See http://wolf.ifj.edu.pl/phonon.

${ }^{36}$ M. M. R. Costa, J. A. Paixão, M. J. M. de Almeida, and L. C. R. Andrade, Acta Crystallogr., Sect. B: Struct. Sci. 49, 591 (1993).

${ }^{37}$ D. L. Lakshtanov, S. V. Sinogeikin, K. D. Litasov, V. B. Prakapenka, H. Hellwig, J. y. Wang, C. Sanches-Valle, J.-P. Perrillat, B. Chen, M. Somayazulu, J. Li, E. Ohtani, and J. D. Bass, Proc. Natl. Acad. Sci. USA 104, 13588 (2007).

${ }^{38}$ J. Haines, J. M. Léger, C. Chateau, and A. S. Pereira, Phys. Chem. Miner. 27, 575 (2000).

${ }^{39}$ S. López-Moreno, A. H. Romero, J. Mejía-López, and A. Muñoz (unpublished).

${ }^{40}$ F. Birch, Phys. Rev. 71, 809 (1947). 
${ }^{41}$ D. Gerlich, S. Hart, and D. Whittal, Phys. Rev. B 29, 2142 (1984).

${ }^{42}$ S. P. S. Porto, P. A. Fleury, and T. C. Damen, Phys. Rev. 154, 522 (1967).

${ }^{43}$ C. Lee and X. Gonze, J. Phys.: Condens. Matter 7, 3693 (1995).

${ }^{44}$ S. S. Rosenblum, W. H. Weber, and B. L. Chamberland, Phys. Rev. B 56, 529 (1997).

${ }^{45}$ B. R. Maddox, C. S. Yoo, D. Kasinathan, W. E. Pickett, and R. T. Scalettar, Phys. Rev. B 73, 144111 (2006).

${ }^{46}$ A. Togo, F. Oba, and I. Tanaka, Phys. Rev. B 78, 134106 (2008).

${ }^{47}$ H. Hellwig, A. F. Goncharov, E. Gregoryanz, H.-k. Mao, and R. J. Hemley, Phys. Rev. B 67, 174110 (2003).

${ }^{48}$ J. Haines, J. M. Léger, C. Chateau, R. Bini, and L. Ulivi, Phys. Rev. B 58, R2909 (1998).

${ }^{49}$ H. Wang, X. Liu, Y. Li, Y. Liu, and Y. Ma, Solid State Commun. 151, 1475 (2011).

${ }^{50}$ K. R. Babu, C. B. Lingam, S. Auluck, S. P. Tewari, and G. Vaitheeswaran, J. Solid State Chem. 184, 343 (2011).
${ }^{51}$ E. Meloche, M. G. Cottam, and D. J. Lockwood, Phys. Rev. B 76, 104406 (2007).

${ }^{52}$ A. Lever, Inorganic Electronic Spectroscopy (Elsevier, Amsterdam, 1984).

${ }^{53}$ I. P. R. Moreira, R. Dovesi, C. Roetti, V. R. Saunders, and R. Orlando, Phys. Rev. B 62, 7816 (2000).

${ }^{54}$ A. Mujica, A. Rubio, A. Muñoz, and R. J. Needs, Rev. Mod. Phys. 75, 863 (2003)

${ }^{55}$ S. Baroni, S. de Gironcoli, A. Dal Corso, and P. Giannozzi, Rev. Mod. Phys. 73, 515 (2001).

${ }^{56}$ V. Panchal, S. López-Moreno, D. Santamaría-Pérez, D. Errandonea, F. J. Manjón, P. Rodríguez-Hernandez, A. Muñoz, S. N. Achary, and A. K. Tyagi, Phys. Rev. B 84, 024111 (2011).

${ }^{57}$ S. López-Moreno and D. Errandonea, Phys. Rev. B 86, 104112 (2012).

${ }^{58}$ W. Haynes, D. Lide, and T. Bruno, CRC Handbook of Chemistry and Physics 2012-2013 (CRC Press, Boca Raton, FL, 2012). 